\title{
On an Algebraic Approach to Higher Dimensional Statistical Mechanics
}

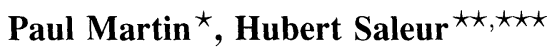 \\ Physics Department, Yale University, New Haven CT06511, USA
}

Received: 15 September 1992

\begin{abstract}
We study representations of Temperley-Lieb algebras associated with the transfer matrix formulation of statistical mechanics on arbitrary lattices. We first discuss a new hyperfinite algebra, the Diagram algebra $D_{n}(Q)$, which is a quotient of the Temperley-Lieb algebra appropriate for Potts models in the mean field case, and in which the algebras appropriate for all transverse lattice shapes $G$ appear as subalgebras. We give the complete structure of this subalgebra in the case $\hat{A}_{n}$ (Potts model on a cylinder). The study of the Full Temperley Lieb algebra of graph $G$ reveals a vast number of infinite sets of inequivalent irreducible representations characterized by one or more (complex) parameters associated to topological effects such as links. We give a complete classification in the $\hat{A}_{n}$ case where the only such effects are loops and twists.
\end{abstract}

\section{Introduction}

Finding integrable statistical mechanics systems in dimension greater than two is notably difficult, and very little is known about that question [1]. In two dimensions there are algebraic structures more general than integrability, whose study nevertheless provides some physical information $[2,3,4]$. These structures are not all constrained to two dimensions. For example the Temperley Lieb [5] algebra: consider the complete unoriented graph of $n$ nodes, here called $\underline{n}$, and all those subgraphs $G \subset \underline{n}$ obtained by removing bonds (edges) from the complete graph.

Definition. 1. We define $T_{G}(Q)$, the Full Temperley-Lieb algebra of the graph $G$ [2], to be the unital algebra over $\mathbb{C}$ with generators

$$
\left\langle 1, U_{i .}(i=1,2, \ldots, n), U_{\imath j}=U_{j i}(\operatorname{edge}(i, j) \in G)\right\rangle
$$

\footnotetext{
* Permanent address: Mathematics Department, City University, Northampton Square, London EC1V 0HB, UK

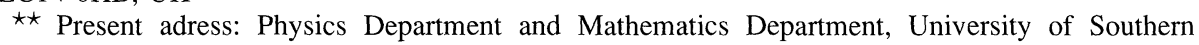
California, Los Angeles CA 90089-0484, USA

$\star \star \star$ Packard Fellow
} 
and relations:

$$
U^{2}=\sqrt{Q} U
$$

(any indices)

$$
\begin{gathered}
U_{\imath .} U_{\imath \jmath} U_{\imath .}=U_{\imath .}, \\
U_{i j} U_{i .} U_{\imath j}=U_{i \jmath}, \\
{\left[U_{i .}, U_{\jmath .}\right]=\left[U_{\imath \jmath}, U_{k l}\right]=\left[U_{i .}, U_{k j}\right]=0, \quad i \neq k, j .}
\end{gathered}
$$

We note the very useful nested structure of inclusions of these algebras (cf. [6]):

$$
G \subset G^{\prime} \Rightarrow T_{G}(Q) \subseteq T_{G^{\prime}}(Q),
$$

where the restriction is achieved by simply omitting the appropriate bond generators. For example, with $G=A_{n}$, the $n$ node chain graph, we recover the original Temperley-Lieb algebra $T_{2 n}(Q)$. Conversely, it follows from the definition of the Potts model [7] that $T_{G}(Q)$ is a generalization of the transfer matrix $(T M)$ algebra $T_{2 n}(Q)=T_{A_{n}}(Q)$ appropriate for building a transfer matrix layer of shape $G$ [2] in other words for overall lattice shape $G \times \mathbb{Z}$. This graph $G$ corresponding to the shape of physical space is not to be confused with the configuration space graphs of $[8,9]$, which work only for the two dimensional case. For example, $G$ a square lattice produces a cubic lattice statistical mechanical model.

For every Temperley Lieb based statistical mechanical model which has a suitable generalization onto a lattice with spacelike layer $G$, such as the Potts model (defined by Hamiltonian

$$
\mathscr{H}=\sum_{(i j) \in G \times \mathbb{Z}} \beta_{i j} \delta_{\sigma_{i} \sigma_{j}},
$$

where $\beta$ is essentially an inverse temperature variable) the transfer matrix algebra provides a representation (abstractly, a quotient) of the Full Temperley Lieb algebra. The inhomogeneous transfer matrix itself is a representation of the element

$$
\tau=\prod_{i=1}^{n}\left(v_{i}+\sqrt{Q} U_{\imath .}\right) \prod_{(i j) \in G}\left(1+\frac{v_{i j}}{\sqrt{Q}} U_{i j}\right)
$$

where $v=\exp (\beta)-1$. The Potts representation is given explicitly in $[2,10]$. By well known arguments $[2,11,12]$ the irreducible representations of $T_{G}(Q)$ which compose this representation are then the most efficient blocks to use in computing the TM spectrum. Moreover in two dimensions $\left(G=A_{n}\right)$, the exceptional cases, where the algebra is not faithfully represented in physical transfer matrices, correspond to models with "rational" conformal field theory limits. A large amount of information about this conformal limit can actually be read in the algebraic properties for finite systems $[9,13]$. By establishing the physically correct generic algebra in other dimensions we develop a procedure for addressing any analogous situation there.

The $G=A_{n}$ algebra is finite dimensional for finite $n$, and typically faithfully represented by the finite dimensional physical transfer matrices. We will show that for general $G$ the Full algebra is always infinite dimensional unless $G=A_{n}$. Since the physical transfer matrices usually remain finite dimensional in higher dimensions (for finite systems) one problem is to find explicitly the finite dimensional quotients of the Full algebra appropriate for these physical systems.

We begin (in the next section) with a discussion of an algebra related to $T_{\underline{n}}$, called the partition algebra $P_{n}(Q)$. It corresponds very closely to the diagram algebra 
$D_{n}$ which is the "mean field limit" of the Potts model. $P_{n}(Q)$ also has subalgebras indexed by a graph, and is one of the easiest cases to analyse, as expected from a "physical" point of view. The algebra $P_{n}(Q)$ provides an organisational link between the physical and abstract algebras we have described. We begin analysing $T_{G}(Q)$ in Sect. 3. We give a complete analysis of the "affine" $\hat{A}_{n}$ case in Sect. 4 . The complications that appear here for the Full algebra, compared to the planar case, may be given a topological interpretation which leads us in Sect. 5 to a topologically motivated analysis of the general case. The classification scheme of representations includes all links that can be embedded in $G \times \mathbb{Z}$. Sundry additional remarks are collected in the last section.

\section{The Partition Algebra}

We now discuss the partition algebra which will play a crucial role in our analysis [14].

\subsection{Partitions of a Set $M$}

First we need to introduce the set $S_{m}$ of partitions of a set $M$ of $m$ distinguished objects

$$
S_{m}=\left\{\begin{array}{l}
\left(\left(M_{1}\right)\left(M_{2}\right) \ldots\left(M_{\imath}\right) \ldots\right): \\
\left.M_{\imath} \subseteq M \text { s.t. } M_{i} \neq \emptyset, \bigcup_{i} M_{\imath}=M, M_{j} \cap M_{k}=\emptyset(j \neq k)\right\} .
\end{array}\right.
$$

For example, if $M$ is the set of the first $m$ natural numbers

$$
\begin{aligned}
S_{2}=\{ & ((12)),((1)(2))\}, \\
S_{4}=\{((1234)),((1)(2)(3)(4)),((123)(4)),((124)(3)), & ((134)(2),((234)(1)),((12)(34)),((13)(24)),((14)(23)), \\
& ((12)(3)(4)),((13)(2)(4)),((14)(2)(3)), \\
& ((23)(1)(4)),((24)(1)(3)),((34)(1)(2))\} .
\end{aligned}
$$

We call the individual equivalenced subsets of the set of objects "parts." Thus $\left(M_{1}\right)=(123)$ is a part of the partition $((123)(4))$, and so on. The set $S_{m}$ is finite for finite $m$. The total dimension is well known $[2,10,15]$

\begin{tabular}{c|ccccccccccccc}
$m$ & 1 & 2 & 3 & 4 & 5 & 6 & 7 & 8 & 9 & 10 & 11 & 12 & 13 \\
\hline$\left|S_{m}\right|$ & 1 & 2 & 5 & 15 & 52 & 203 & 877 & 4140 & 21147 & 115975 & 678570 & 4213597 & 27644437
\end{tabular}

We write $i \sim^{A} j$ in case objects $i, j$ are in the same partition in $A \in S_{m}$, so $\sim^{A}$ is transitive.

We will be mainly interested in the case $m=2 n$. We will then write our $2 n$ objects simply as

$$
1,2,3, \ldots, n, 1^{\prime}, 2^{\prime}, 3^{\prime}, \ldots, n^{\prime}
$$


2.2. Operations on $S_{m}(m=2 n)$

For $Q$ an indeterminate and $K$ the field of rational functions of $Q$ we define a product $[2,10]$

$$
\begin{gathered}
\mathscr{P}: S_{m} \times S_{m} \rightarrow K S_{m}, \\
(A, B) \mapsto A B=Q^{f(A, B)} C
\end{gathered}
$$

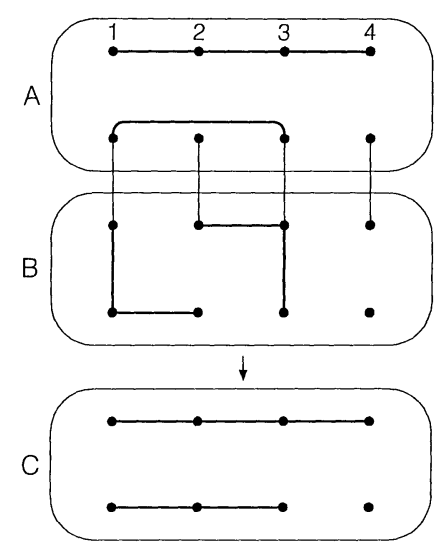

Fig. 1. Pictorial realisation of parts as clusters and composition of partitions by juxtaposing clusters. In this case $f((A, B))=0$

Where $C \in S_{m}$ and $f(A, B) \in \mathbb{Z}_{>0}$ are defined as follows. Relabel the objects in $B$ from

$$
1^{\prime \prime}, 2^{\prime \prime}, \ldots, n^{\prime \prime}, 1^{\prime \prime \prime}, 2^{\prime \prime \prime}, \ldots, n^{\prime \prime \prime}
$$

Form a partition of $4 n$ objects from $A$ and $B$ by first taking the parts in $A$ and including into the part containing $i^{\prime}$ the part from $B$ containing $i^{\prime \prime}$. Then delete all the $i^{\prime}$ and $i^{\prime \prime}$ [counting the number $f(A, B)$ of parts which become empty, and are then discarded, in the process] and finally relabel all the $i^{\prime \prime \prime}$ as $i^{\prime}$. The partition of $2 n$ objects obtained is $C$.

For example,

$$
\begin{aligned}
& \left((1234)\left(1^{\prime} 3^{\prime}\right)\left(2^{\prime}\right)\left(4^{\prime}\right)\right)\left(\left(11^{\prime} 2^{\prime}\right)\left(233^{\prime}\right)\left(44^{\prime}\right)\right) \\
& \quad \rightarrow\left((1234)\left(1^{\prime} 3^{\prime} 1^{\prime \prime} 1^{\prime \prime \prime} 2^{\prime \prime \prime} 2^{\prime \prime} 3^{\prime \prime} 3^{\prime \prime \prime} 2^{\prime}\right)\left(4^{\prime} 4^{\prime \prime} 4^{\prime \prime \prime}\right)\right) \\
& \rightarrow\left((1234)\left(1^{\prime \prime \prime} 2^{\prime \prime \prime} 3^{\prime \prime \prime}\right)\left(4^{\prime \prime \prime}\right)\right) \rightarrow\left((1234)\left(1^{\prime} 2^{\prime} 3^{\prime}\right)\left(4^{\prime}\right)\right)
\end{aligned}
$$

This is illustrated in Fig. 2.2. There are other such illustrations in Sect. 2.5.

Definition 2 (Partition algebra [14]). Considering the vector space over $K$ spanned by $S_{2 n}$, the linear extension of the product $\mathscr{P}$ gives us a finite dimensional algebra over $K$ which we call the partition algebra $P_{n}(Q)$.

Definition 3. The natural inclusion $\mathscr{S}$ is defined by

$$
\begin{gathered}
0 \rightarrow P_{n-1} \stackrel{\mathscr{\mathscr { S }}}{\longrightarrow} P_{n}, \\
\mathscr{S}:((\ldots) \ldots(\ldots)) \mapsto\left((\ldots) \ldots(\ldots)\left(n n^{\prime}\right)\right) .
\end{gathered}
$$




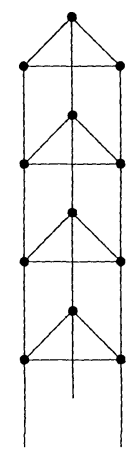

Fig. 2. Part of the graph $\underline{3} \times \mathbb{Z}$

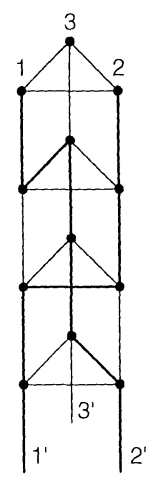

Fig. 3. Diagram for the connectivity $1_{12} U_{3}=\left(\left(12^{\prime}\right)\left(21^{\prime}\right)(3)\left(3^{\prime}\right)\right)$ which restricts to $1_{12}$ for $n=2$

It is convenient to introduce the following special elements of the partition algebra:

$$
\begin{gathered}
1=\left(\left(11^{\prime}\right)\left(22^{\prime}\right) \ldots\left(n n^{\prime}\right)\right), \\
1_{\imath \jmath}=\left(\left(11^{\prime}\right)\left(22^{\prime}\right) \ldots\left(i j^{\prime}\right) \ldots\left(j i^{\prime}\right) \ldots\left(n n^{\prime}\right)\right), \quad i, j=1,2, \ldots, n, \\
A_{\imath .}=\frac{1}{\sqrt{Q}}\left(\left(11^{\prime}\right)\left(22^{\prime}\right) \ldots(i)\left(i^{\prime}\right) \ldots\left(n n^{\prime}\right)\right), \\
A_{i \jmath}=\sqrt{Q}\left(\left(11^{\prime}\right)\left(22^{\prime}\right) \ldots\left(i j i^{\prime} j^{\prime}\right) \ldots\left(n n^{\prime}\right)\right) .
\end{gathered}
$$

Proposition 1 (see [14]). These elements generate $P_{n}(Q)$.

Definition. 4. For $A \in P_{n}$ let [A] denote the maximum over the $S_{m}$ components of $A$ of the number of distinct parts containing both primed and unprimed elements.

For example $[1]=n,\left[A_{i}\right]=n-1$. Then

Corollary 1.1. For $A, B \in P_{n}$,

$$
[A B] \leq \min ([A],[B])
$$


Proposition 2. There is a homomorphism from the Full Temperley-Lieb algebra of the complete graph $\underline{n}$ to the partition algebra given by

$$
\begin{aligned}
H: T_{\underline{n}}(Q) & \rightarrow P_{n}(Q), \\
H: 1 & \mapsto 1, \\
H: U_{i .} & \mapsto A_{i}, \\
H: U_{i j} & \mapsto A_{i j} .
\end{aligned}
$$

Proposition 3 (see [2]). The subalgebra of $P_{n}(Q)$ generated by

$$
\left\langle 1, A_{i .}(i=1,2, \ldots, n), A_{i \imath+1}(i=1,2, \ldots, n-1)\right\rangle
$$

is isomorphic to $T_{A_{n}}(Q)$.

Definition 5. For given $n$ we define $\Sigma_{n}$ as the subalgebra of $P_{n}(Q)$ generated by

$$
\left\langle 1,1_{\imath j}(i, j=1,2, \ldots, n)\right\rangle
$$

or, where appropriate, as the corresponding symmetric group.

\subsection{Full Embedding of $P_{n-1}$ in $P_{n}$}

We will need the following simple but surprisingly powerful theorem:

Theorem 1 (see [14]). For each $n, Q \neq 0$ and idempotent $e=e_{n}=A_{n} / \sqrt{Q}$ there is an isomorphism of algebras

$$
e_{n} P_{n} e_{n} \cong P_{n-1}
$$

As a consequence the categories of left $P_{n-1}$ and left $P_{n} e_{n} P_{n}$ modules are essentially isomorphic categories (the extend to which they are not isomorphic is, for our purposes, a technicality - the interested reader should turn to $[14,16,18]$ for details).

Let us denote by $F_{n}(M)=e_{n} M$ the object map from $\left(P_{n}-\bmod \right)$ to $\left(P_{n-1}-\bmod \right)$ at level $n$.

Corollary 1.1 (see [14]). Let $f_{n}$ be the object map of categories defined by restriction of left $P_{n}$ modules to left $P_{n-1}$ modules through the inclusion $\mathscr{S}$,

$$
\begin{gathered}
f_{n}:\left(P_{n}-\bmod \right) \rightarrow\left(P_{n-1}-\bmod \right), \\
f_{n}: M \mapsto P_{n-1} \downarrow M .
\end{gathered}
$$

Then the following diagram of object maps of categories commutes:

$$
\begin{gathered}
\left(P_{n}-\bmod \right) \stackrel{F_{n}}{\longrightarrow}\left(P_{n-1}-\bmod \right) \\
f_{n} \downarrow \\
\left(P_{n-1}-\bmod \right) \stackrel{F_{n-1}}{\longrightarrow}\left(P_{n-2}-\bmod \right)
\end{gathered}
$$

This implies that, up to edge effects caused by the difference between $P_{n}$ and $P_{n} e_{n} P_{n}$, the Bratteli restriction diagram for the algebras $P_{n}$ (see Sect. 2.6.3 onwards) has the same structure on each level $n$. But then 
Proposition 4 (see [14]). The following is a short exact sequence of algebras

$$
0 \rightarrow P_{n} e P_{n} \rightarrow P_{n} \rightarrow \Sigma_{n} \rightarrow 0 .
$$

Thus, at least for $P_{n}$ semi-simple, a knowledge of the structure of $P_{n-1}$ essentially determines for us the structure of $P_{n}$.

Corollary 4.1. In case $P_{n}(Q)$ semi-simple the distinct equivalence classes of irreducible representations may be indexed by the list of all standard partitions of every integer from 0 (understood to have one standard partition) to $n$.

In fact $P_{n}(Q)$ is semi-simple for $Q$ indeterminate and for all $Q \in \mathbb{C}$ except for the roots of a finite order polynomial in $Q$ for any finite $n$ (see Sect. 6).

\subsection{Diagram Algebra for a Graph $G$}

Let us return to Proposition 3. More generally we have

Definition 6. For graph $G$ the Diagram algebra $D_{G}(Q)$ is defined as the subalgebra of the partition algebra generated by

$$
\left\langle 1, A_{\imath .}(i=1,2, \ldots, n), A_{i j}(i, j \in G)\right\rangle .
$$

Note that $D_{\underline{n}}(Q) \subset P_{n}(Q)$, as $1_{\imath \jmath}$ cannot be built with these generators. However, under certain conditions it can be substituted, for example,

$$
1_{23} A_{1}=A_{1} . A_{12} A_{2 .} A_{23} A_{3}, A_{13} A_{1} .
$$

In fact we are more interested here in $D_{\underline{n}}(Q)$ than $P_{n}(Q)$ (compare Proposition 2 with Eq. 6), but $P_{n}(Q)$ provides a more versatile general setting. We will see shortly that it is straightforward to move from one to the other.

The relationship between the algebra types $T, P$, and $D$ is summarized by the commutative diagram

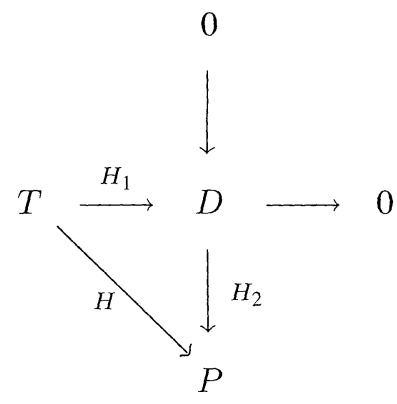

which is exact at $D$.

Proposition 5. The subalgebra $D_{n}(Q) \subset P_{n}(Q)$ is invariant under conjugation by elements of the group $\Sigma_{n}$, i.e.

$$
b^{-1} D_{\underline{n}}(Q) b=D_{\underline{n}}(Q) \quad \forall b \in \Sigma_{n} .
$$

Corollary 5.1. Every word in $P_{n}(Q)$ can be written in the form $A B$ where $A \in \Sigma_{n}$ and $B \in D_{\underline{n}}(Q)$. 
Clearly we have the rich inclusion structure again

$$
G \supset G^{\prime} \Rightarrow D_{G}(Q) \supseteq D_{G^{\prime}}(Q) \text {. }
$$

It also follows that $D_{G}(Q)$, and indeed $P_{n}(Q)$, obeys a number of quotient relations in addition to the Temperley-Lieb relations. For example, with $W \in D_{G}(Q)$ there exists $X(W)$ a certain (known) scalar function of $Q$ (see [2]) such that

$$
\left(\prod_{i} A_{\imath}\right) W\left(\prod_{i} A_{i .}\right)=X(W)\left(\prod_{i} A_{i .}\right) \text {. }
$$

Specifically, if $W \in S_{m}$ with $b_{W}$ parts

$$
X(W)=Q^{b_{W}} .
$$

This relation is suitable for at least part of the set appropriate for physical systems, as it corresponds to the existence of disorder at very high temperatures (there is also a dual corresponding to order at low temperatures). At the level of the dichromatic polynomial it corresponds to isolating $b_{W}$ clusters (cf. [7], for example). Several analogous relations have also been found [2].

\subsection{Pictorial Realisation by Connectivities}

Definition 7. For a graph $G$ let $\mathscr{B}_{G}$ be the universal set of the set of bonds of $G$, i.e. the set of all (not necessarily proper) subgraphs of $G$ of order $|G|$ nodes (obtained by omitting bonds).

Note that elements of $\mathscr{P}_{G}$ may not be connected graphs $[7,17]$.

The partitions $A \in D_{\underline{n}}(Q) \cap S_{m}$ may be realised as classes of $\mathscr{B}_{\underline{n} \times \mathbb{Z}}$ under a certain equivalence $\varrho$. The construction of $\varrho$ is as follows.

Explicitly number the nodes of $\underline{n}$ at "time" $t=0$ from $1,2, \ldots, n$. Practically it will be convenient to restrict attention in $\mathscr{B}_{\underline{n} \times \mathbb{Z}}$ to the subset of elements which for sufficiently large $t$ have all time-like bonds present and all space-like bonds absent. This is a sort of very large time asymptotic smoothness condition. For some such very large $t=T$ number the nodes of $(\underline{n}, T)$ from $1^{\prime}, 2^{\prime}, \ldots, n^{\prime}$. Then introduce the map

$$
\begin{gathered}
F_{T}: \mathscr{P}_{\underline{n} \times \mathbb{Z}} \rightarrow P_{n}(Q), \\
F_{T}: B_{0} \mapsto Q^{b} B,
\end{gathered}
$$

where $B \in S_{m}$ such that $i \sim^{B} j$ iff $i, j$ are connected by a path of bonds present in the subgraph $B_{0}$, and $b$ is the number of isolated connected components in $B_{0}$ not connected to any point in either of the layers $t=0$ or $t=T$.

The point about the limits $t=0, T$ is that for finite $n$ there exists some finite $T$ beyond which (range $\left.F_{T}\right) \cap S_{m}$ does not increase. Thus the asymptotic condition is not important (just convenient), but ensures that $F_{T}$ and $F_{T+1}$ are essentially the same map.

The equivalence classes of $\mathscr{B}_{\underline{n} \times \mathbb{Z}}$ are defined so as to make this map an injection (i.e. $B_{0} \varrho C_{0}$ only if $B=C$ ).

The range of $F_{T}$ does not include the whole of $S_{m}$ however large we make $T$ (see the remark after Definition 1). We can extend to the whole of $S_{m}$ by, for example, building our "connectivities" on $\underline{n+1} \times \mathbb{Z}$ (but only labelling the "first" $n$ nodes). This complication is connected to the nature of the lattice and the TM formalism; 
it will be discussed further elsewhere. In general, different choices of $G$ in $\mathscr{B}_{G \times \mathbb{Z}}$, realise different sets of connectivities, i.e. different ranges for $F_{T}$. This is, in fact, the essence of the physically important problem of finding irreducible representations of $D_{G}(Q)$ (see later).

We may extend $\mathscr{B}_{n+1 \times \mathbb{Z}} / \varrho$ or $\mathscr{B}_{\underline{n} \times \mathbb{Z}} / \varrho$ to an algebra (over rational functions in $Q$ ) by defining a product $\bar{B}_{0} C_{0}$. We first build a new graph $(B C)_{0}$ by discarding $t>T$ in $B_{0}$ and $t<0$ in $C_{0}$ and the joining $B_{0}$ and $C_{0}$ by identifying the layer $t=T$ in $B_{0}$ with $t=0$ in $C_{0}$. Let $D \in S_{m}$ have the same connectivities as the graph so produced has between $t=0$ and $t=2 T-1$ [i.e. $F_{2 T-1}\left((B C)_{0}\right)=Q^{d} D$ for some $d]$. We then define $B_{0} C_{0}=Q^{d} D_{0}$, where $D_{o} \in \mathscr{B} / \varrho$ is such that $F\left(D_{0}\right)=D$. The map $F$ is then an algebra homomorphism.

The explicit pictorial realization is particularly neat (but sufficiently general) if we distribute the nodes of $\underline{n}$ linearly, as in $A_{n}$, and only draw the part of the graph not in the asymptotic region. Then for example with $n=12$ the $\varrho$ class of $A_{i i+1}$ has a simple representative

$$
A_{i+1} / \sqrt{Q} \leftarrow \bullet \bullet \bullet \bullet \bullet \bullet \bullet \bullet \bullet \bullet .
$$

The $\varrho$ class of $A_{i}$. has representative

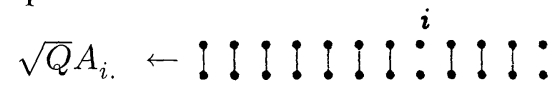

The composition rule is to identify the top row of dots in the second diagram with the bottom row in the first. Clusters then isolated from both top and bottom rows of the new diagram so formed may be removed, contributing a factor $Q$.

Finally, then, for example, the TL relation 2

$$
A_{i i+1} A_{i .} A_{i i+1}=A_{\imath \imath+1}
$$

amounts to the statement that the subgraph

$$
\text { !!!!! : }
$$

has the same list of connections within and between the top and bottom layers as the $\varrho$ representative of $A_{i+1}$ above.

Note that no composition of diagrams increases the number of distinct connected clusters connecting between the top and bottom layers. This means that the subset of $\varrho$ cosets with no connections top to bottom form a basis for a $P_{n}(Q)$ bimodule. Furthermore, the subset with $\leq p$ distinct connections top to bottom also form a basis for a $P_{n}(Q)$ bimodule.

\subsection{Structure and Representation Theory of $P_{n}(Q)$}

This picture is particularly useful for constructing representations. The number of distinct connections running from $t=0$ to $t=T$ is evidently non-increasing in any composition (it is a measure of the number of distinct bits of information which can be simultaneously propagated through the bond covering, which cannot exceed the number propagated across any fixed time slice). So for example, writing simply $P$ or $P_{n}$ for $P_{n}(Q)$, and defining idempotents

$$
I_{k}=\prod_{i>k} \frac{H\left(U_{i .}\right)}{\sqrt{Q}}
$$


$(Q \neq 0)$ then $I_{0}$ allows no connections from $t=0$ to $t=T$, so $P_{n} I_{0} P_{n}$ is the invariant subspace of $P_{n}$, where

$$
\nexists A, i, j \quad \text { s.t. } \quad i \sim^{A} j^{\prime} .
$$

Proposition 6. The element $I_{0}$ is a primitive idempotent.

Corollary 6.1. The left ideal $P_{n} I_{0}$ is indecomposable (and generically simple).

Note that $\operatorname{dim}\left(P_{n} I_{0}\right)=\left|S_{n}\right|$.

Proposition 7. The element $I_{1}$ is primitive in the quotient algebra $P_{n} / P_{n} I_{0} P_{n}$, so again $P_{n} I_{1}$ is indecomposable in this quotient.

Now $I_{2}$ is not primitive in $P_{n} / P_{n} I_{1} P_{n}$ since, for example

$$
I_{2} 1_{12} I_{2} \sim 1_{12} I_{2} \not \subset I_{2} \text {. }
$$

On the other hand $\frac{\left(1+1_{12}\right)}{2} I_{2}$ and $\frac{\left(1-1_{12}\right)}{2} I_{2}$ are primitive idempotents.

Similarly $I_{3}$ is not primitive in $P_{n} / P_{n} I_{2} P_{n}$, but, for example

$$
\Sigma_{ \pm} I_{3}=\frac{\left(1 \pm 1_{12} \pm 1_{23} \pm 1_{13}+1_{12} 1_{23}+1_{13} 1_{23}\right)}{3 !} I_{3}
$$

and two further combinations (with other symmetries) are.

From the definition of $I_{\imath}$ we have $P_{n} I_{i-1} P_{n} \subset P_{n} I_{i} P_{n}$ and a nest of short exact sequences, $i=1,2, \ldots, n$,

$$
0 \rightarrow P_{n} I_{\imath-1} P_{n} \rightarrow P_{n} I_{i} P_{n} \rightarrow P_{n} I_{i} P_{n} / P_{n} I_{i-1} P_{n} \rightarrow 0
$$

where finally $I_{n}=1$.

Definition 8. Let us define the algebra $P_{n}[i]=P_{n} I_{\imath} P_{n} / P_{n} I_{i-1} P_{n}$.

This is the algebra of elements with not more than $i$ distinct connections running, as it were, from $t=0$ to $t=T$, quotiented by the invariant subspace of all elements with strictly less than $i$ distinct connections from 0 to $T$.

Proposition 8. In the quotient $P_{n}[i]$,

$$
I_{i} \Sigma_{n} I_{i}=\Sigma_{i} I_{\imath}
$$

(we take $\Sigma_{0}=\Sigma_{1}=1$ ).

Proposition 9. Let $\Sigma$ be any left $\Sigma_{\imath}$ module. Then we can write the left $P_{n}[i]$ module,

$$
P_{n}(Q)\left(I_{i} \Sigma\right)=D_{\underline{n}}(Q)\left(I_{i} \Sigma\right) .
$$

2.6.1. To Construct Irreducible Representations. For each $i=0, \ldots, n$ and $\lambda \vdash i(\lambda$ a partition of $i$ ) and $\Sigma_{\lambda}$ the $\lambda$ simple $\Sigma_{i}$ module [20], the set $S_{m} I_{i} \Sigma_{\lambda}$ generates a basis for a generic irreducible representation.

Let us first consider the fully symmetrized case for the left $\Sigma_{i}$ module, call it $\Sigma^{\prime}$, in each sector $i$. Then we get a basis for the left $P_{n}[i]$ module $P_{n} I_{i} \Sigma^{s}$ from $S_{m}$ as follows. List the elements as partitions of $1,2, \ldots, n$, ignoring $1^{\prime}, 2^{\prime}, \ldots, n^{\prime}$ except in so far as to note which parts originally contained primed elements. We discard duplicate copies of partitions not distinguished by this property, and partitions in 
which other than $i$ parts originally contained primed elements. We call the resultant set $S_{n}(i)$. For example,

$$
S_{2}(1)=\left\{\left((12)^{\prime}\right),\left((1)^{\prime}(2)\right),\left((1)(2)^{\prime}\right)\right\} .
$$

We do not need to keep track of exactly which unprimed nodes were connected to which primed nodes, since the symmetriser makes all these permutations equivalent. In other words the set $S_{n}(i)$ is the set of all possible ways of arranging the elements of $S_{n}$ (cf. $S_{m}=S_{2 n}$ ) so that $i$ parts are distinguished from the rest. An element of $S_{n}$ with $p \geq i$ parts produces $p !((p-i) ! i !)$ elements of the basis $S_{n}(i)$ (and produces none if $p<i$ ). Note that

$$
\sum_{i=0}^{n} S_{n}(i)=2^{n} S_{n} .
$$

The action of the generators on such a basis is just the usual product (7) (remembering the $P_{n}[i]$ quotient). It is given explicitly in $[2,10]$. We will prove irreducibility of these representations in Sect. 2.6.3.

Moving to the case where we take some other left $\Sigma_{\imath}$ module in Proposition 9, then our basis must simply be (semi) direct producted with a basis for this new module. Permuting actions will act on the $\Sigma_{i}$ module rather than the partitions.

2.6.2. The Case $n=3$. We can well illustrate all of these points with an example. Let us consider $n=3$. The available partition shapes $\lambda$ in $S_{6}$ are:

$$
(6),(5,1),(4,2),\left(3^{2}\right),\left(4,1^{2}\right),(3,2,1),\left(2^{3}\right),\left(3,1^{3}\right),\left(2^{2}, 1^{2}\right),\left(2,1^{4}\right),\left(1^{6}\right)
$$

with corresponding multiplicities $\mathscr{V}_{\lambda}$ :

$$
1,6,15,10,15,60,15,20,45,15,1 \text {, }
$$

giving total dimension $\left|S_{6}\right|=203$.

On the other hand the dimensions of the bases described above are

$$
5,10,6 \operatorname{dim}\left(\Sigma_{2}\right), 1 \operatorname{dim}\left(\Sigma_{3}\right)
$$

i.e., explicitly, the bases are (with parts after the colon understood primed)

$$
\begin{gathered}
\{((123): \emptyset),((12)(3): \emptyset),((13)(2): \emptyset),((23)(1): \emptyset),((1)(2)(3): \emptyset)\}, \\
\{(\emptyset:(123)),((12):(3))((13):(12)),((13):(2)),((2):(13)), \\
((23):(1)),((1):(23)),((1)(2):(3)),((1)(3):(2)),((2)(3):(1))\}, \\
\{(\emptyset:(12)(3)),(\emptyset:(23)(1)),(\emptyset:(2)(13)),((1):(2)(3)), \\
((2):(1)(3)),((3):(1)(2))\} \times \Sigma_{ \pm} \\
\{(\emptyset:(1)(2)(3))\} \times \Sigma_{3} .
\end{gathered}
$$

Finally, then, noting the multiplicities of inequivalent generically irreducible representations at level $i$ we have

$$
5^{2}+10^{2}+6^{2} \cdot(1+1)+1^{2} \cdot\left(1+2^{2}+1\right)=203=\left|S_{6}\right|,
$$

so we have, for example, the complete set of inequivalent irreducible representations for the semisimple cases. Note that all the $i=3$ representations reduce to (direct sums of) the same representation in $D_{n}(Q)$, because none of the permutations can actually be realized in this subalgebra. 
2.6.3. General $n$. Since we know the structure of the symmetric group (algebra) $\Sigma_{i}$ (see, for example, $[19,20]$ ) it behooves us to divide up our analysis by first considering the algebra for the $\Sigma$-symmetrised case, $P_{n}(Q) / \sim$, which we define below. The rest then follows from changing the left $\Sigma_{i}$ module in Proposition 9.

Definition 9. We define an equivalence relation $\sim$ on $S_{m}$ by $A \sim B$ iff they are the same up to a permutation of the connections made by the connectivities from $t=0$ to $t=T$.

We write $P_{n}(Q) / \sim$ for the quotient algebra obtained by the linear extension to $P_{n}(Q)$.

In this case there is a bra-ket notation for elements of $S_{m}$. Every element may be written uniquely in the form $|a\rangle\langle b|$, where $a, b \in S_{n}(i)$ for some $i$ (conversely every such pair defines a unique element). There is then an obvious inner product $\langle b \mid c\rangle$ in each $P_{n}[i] / \sim$, obtained from

$$
|a\rangle\langle b|| c\rangle\langle d|=\langle b \mid c\rangle| a\rangle\langle d|
$$

Proposition 10. The $n+1$ representations of $P_{n}(Q) / \sim$ with bases $S_{n}(i)(i=$ $0,1,2, \ldots, n$ ) and canonical action (up to the $P_{n} I_{\imath-1} P_{n}$ quotient) are each irreducible for $Q$ indeterminate.

Corollary 10.1. These representations are inequivalent.

Corollary 10.2. Any representation of $P_{n}(Q)$ built from Proposition 9 with $\Sigma$ an irreducible $\Sigma_{i}$ module is irreducible.

Corollary 10.3. $P_{n}(Q)$ is semi-simple for $Q$ indeterminate and for all $Q \in \mathbb{C}$ except for the roots of a finite order polynomial in $Q$ for any finite $n$.

Proof. By dimension counting. The irreducible representations account for the full dimension of the algebra. We can show this explicitly as follows:

The Bratelli diagram for the restriction corresponding to

$$
\left(P_{n}(Q) / \sim\right) \supset\left(P_{n-1}(Q) / \sim\right)
$$

on these irreducible representations is as follows (with top line $n=0$ )

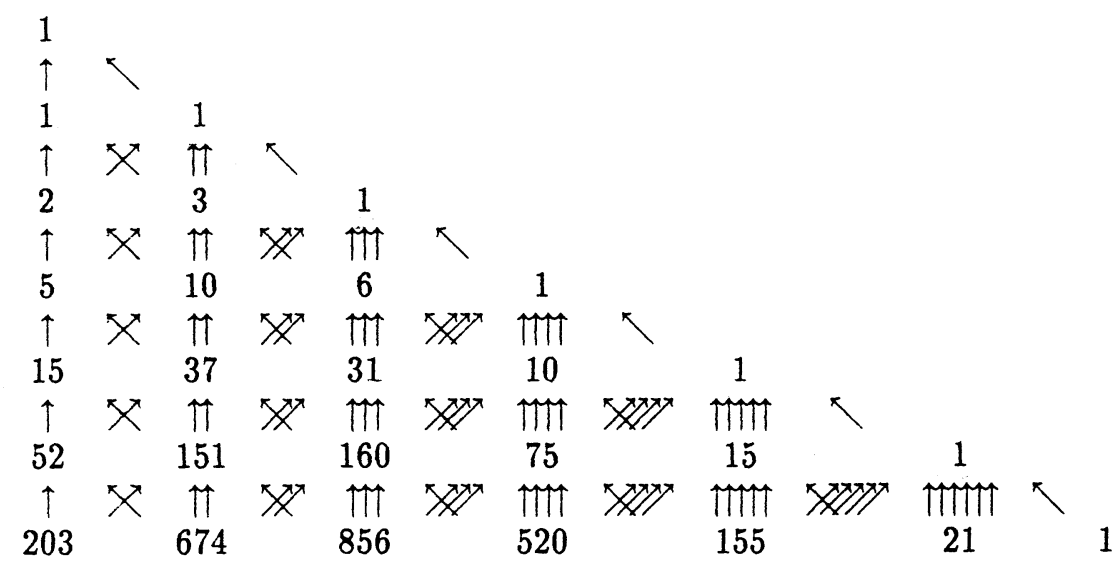


and so on. These restrictions are forced by a Morita equivalence theorem - cf. [2]. This works as follows. The isomorphism of categories in the corollary to Theorem 1 takes a layer of the above diagram to the layer below it (each node is mapped vertically down, since the idempotent $e_{n}$ cuts at most one connection). The 1 at the right-hand side of the lower layer is missing, of course, as this is the trivial representation of $\Sigma_{n}$. Consequently (i.e. as a knock on effect from the previous layer) the restriction information for the next two modules to the left is incomplete. However, the only possibility is for the restrictions to include some copies of the trivial representation, and these may be filled in by dimension counting. For example, omitting node 3 in $S_{3}(2)$ we get

$$
\begin{aligned}
& \left\{\left((1)^{\prime}(23)^{\prime}\right),\left((12)^{\prime}(3)^{\prime}\right),\left((13)^{\prime}(2)^{\prime}\right),\left((1)^{\prime}(2)^{\prime}(3)\right),\left((1)^{\prime}(2)(3)^{\prime}\right),\left((1)(2)^{\prime}(3)^{\prime}\right)\right\} \\
& \quad \rightarrow\left\{\left((1)^{\prime}(2)^{\prime}\right),\left((12)^{\prime}\right),\left((1)^{\prime}(2)^{\prime}\right),\left((1)^{\prime}(2)^{\prime}\right),\left((1)^{\prime}(2)\right),\left((1)(2)^{\prime}\right)\right\} \\
& =S_{2}(1)+3 . S_{2}(2) .
\end{aligned}
$$

Note that in omitting the last node $(n)$ in this mnemonic if we have a part of the form $(i j \ldots m n)$ (i.e. unprimed) then this maps to $(i j \ldots m)^{\prime}$, since the action of generators here is as if the part is connected to something!

Irreducible representations of $P_{n}(Q)$ follow by Corollary 10.2.

Let us write $d_{n}(i)$ for the dimension of the $i^{\text {th }}$ representation in row $n$ (the $i^{\text {th }}$ column, counting the left-hand column as column 0 ). Then the total dimension of $P_{n}(Q)$ is

$$
\left|S_{m}\right|=\sum_{i=0}^{n}(i) !\left(d_{n}(i)\right)^{2}=\left|S_{m}(0)\right|
$$

when $m=2 n$ as required (the last identity is readily proved). Altogether $S_{m}(i) \times$ $\Sigma_{\lambda}(\lambda \vdash i)$ gives the complete structure for all semi-simple cases.

\section{3. $D_{G}$ for Arbitrary Graphs $G$}

\subsection{The Structure of $D_{\underline{n}}(Q)$}

The structure of the $D_{\underline{n}}$ algebra is very similar to $P_{n}(Q)$. For $P_{n}$ each node in column $i$ of the Bratelli diagram above represents a list of irreducibles, one for each partition of $i$ (with dimension multiplied by the dimension of the corresponding representation of the symmetric group). The only difference here is that the $i=n$ representation (the rightmost one on each line) gives only a single (one dimensional) representation. This just corresponds to the impossibility of any transverse movement of $n$ distinct connected lines on a graph with only $n$ nodes in the lateral direction.

\subsection{On the Structure of $D_{G}$}

Proposition 11. The connectivities which can be realised on $G \times \mathbb{Z}$ provide bases for the irreducible representations of $D_{G}$.

This follows since in $D_{G}$ these subsets span invariant subspaces. Computing the dimension is more difficult. See Sect. 6.

We will find in Sect. 4 that the above finite dimensional algebras do not include all those appropriate for systems of interest in physics. 
Two important questions arise:

1. How do the finite dimensional algebras $D_{G}$ fit into the overall structure of $T_{G}$ ? (see Sect. 4).

2. What is the asymptotic growth rate of dimensions of representations corresponding to a given physical observable or more generally, which $Q$ values are exceptional for each physical dimension? This is also answered in some cases in Sect. 4.

\subsection{Review of $G=A_{n}$}

Consider a twice punctured sphere. Put the unprimed elements of $M$ in natural order at intervals around one boundary, and the primed ones opposite them around the other boundary. Draw a "seam" from one boundary to the other on the sphere, beginning between 1 and $n$ and ending between $1^{\prime}$ and $n^{\prime}$. A partition $A \in S_{m}$ is accessed from $T_{A_{n}}(Q)$ [i.e. in $H\left(T_{A_{n}}(Q)\right)$ ] iff for every part in $A$ a line can be drawn on the sphere connecting all the elements of the part, but touching no other lines, and not touching the seam.

If the connecting lines are thickened into ribbons then the usual boundary diagrams $[2,18,21]$ - Fig. 4 and so on, in case $n=6$ - represent the edges of these lines. Representatives of all equivalence classes under continuous deformation of non-overlapping segments are realized.

A boundary diagrammatic representation of words in $D_{A_{n}}(Q)$ is obtained as follows. The generator $U_{\imath}$ maps to the coset of the diagram shown in Fig. 4. Generators are then composed by connecting the bottom of the diagram for the first factor with the top of the diagram for the second, so

$$
U_{i} U_{i+1} U_{i}=U_{\imath}
$$

is given by Fig. 5. In such diagrams we simply pull the line starting in the $i+2$ position straight to exhibit the identity.

Similarly $U_{i} U_{i}=\sqrt{Q} U_{i}$ is represented in Fig. 6. Here we must interpret closed loops as removable, after contributing a factor $\sqrt{Q}$. These diagrams can then be used to match any word to a reduced word (one which cannot be shortened using the relations), with an appropriate scalar factor.

Note that here

$$
I_{0}=\prod_{\imath} U_{\imath} / \sqrt{Q}
$$

$(Q \neq 0)$ is a primitive idempotent. Writing simple $T$ for $T_{A_{n}}(Q)$ the left ideal $T I_{0}$ is thus indecomposable (and hence generically irreducible). Let us define

$$
I_{k}=\prod_{i>k} \frac{U_{i}}{\sqrt{Q}}
$$




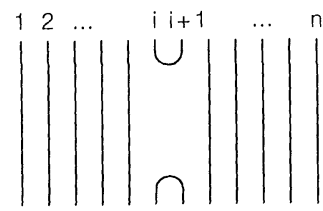

Fig. 4. Boundary diagram for $U_{\imath}$ in $D_{A_{n}}(Q)$, cf. $A_{\jmath} .(j=(i+1) / 2)$ in the connectivity picture



Fig. 5. Boundary diagram exhibiting the relation $U_{\imath} U_{\imath+1} U_{i}=U_{\imath}$

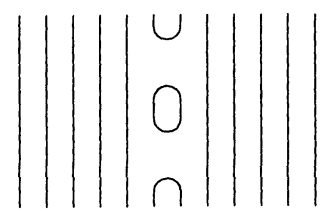

Fig. 6. Diagram for $U_{\imath} U_{\imath}$ before removing the closed loop

On quotienting by the double sided ideal $T I_{0} T$ we find that $I_{1}$ becomes a primitive idempotent, so $T I_{1}$ is indecomposable in $T / T I_{0} T$, and so on. Iterating this filtering process with respect to $k$ we generate bases for all $n+1$ generically irreducible representations. The dimensions of these representations are then readily determined by reference to the tower relation

$$
A_{n} \subset A_{n+1} \Rightarrow T_{A_{n}}(Q) \subset T_{A_{n+1}}(Q)
$$

The argument is precisely analogous to that of Sect. 2.3 (an almost identical construction gives essentially a Morita equivalence $T_{A_{n-1}} \sim T_{A_{n}} e_{n} T_{A_{n}}$, and so on). Extended concrete examples for the $A_{n}$ case in particular are given in Sect. 9.5.2 of reference [2].

It follows (cf. Sect. 2.6.3 - but in this case we make use of the symmetry under reversing the order of the chain, i.e. swapping $e_{1}$ and $e_{n}$ ) that the generic Bratteli restriction diagram for the irreducible representations associated to $T_{n}(Q) \supset T_{n-1}(Q)$ (with top line $n=0$ ) is: 


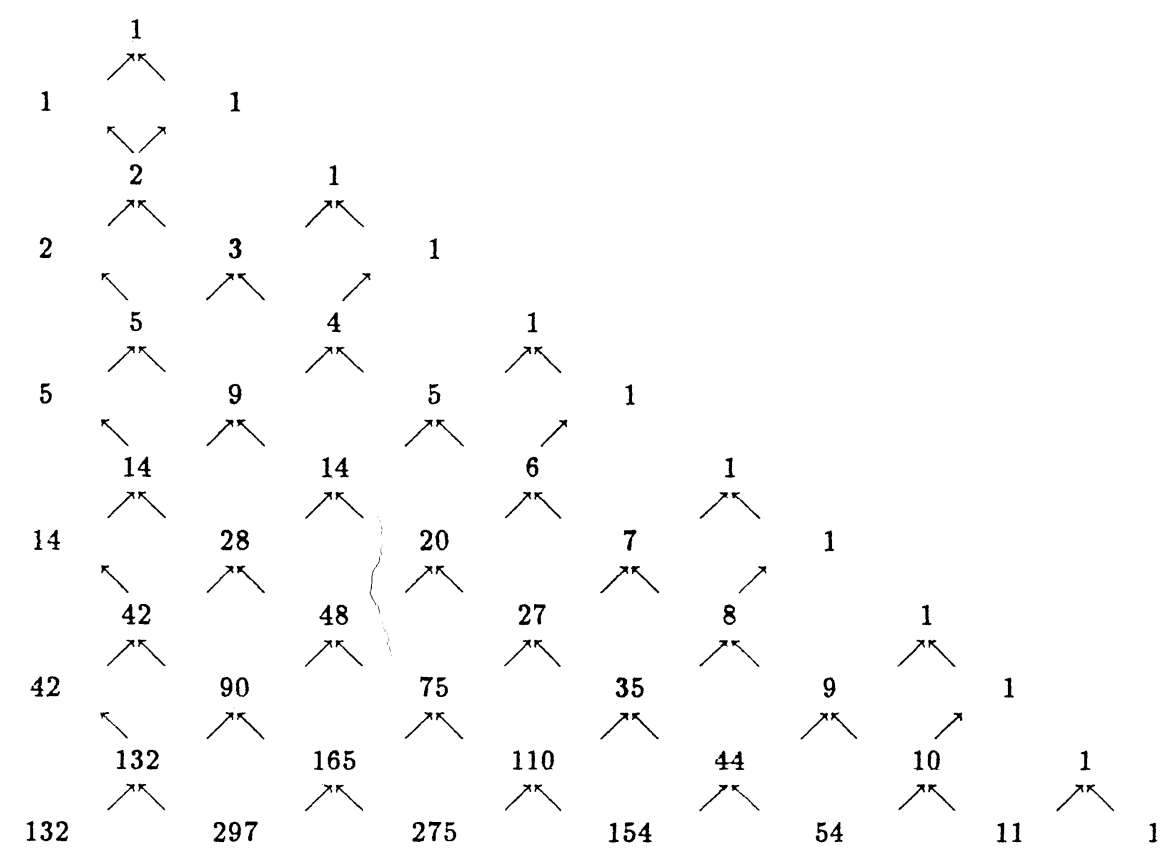

and so on. Recall, for comparison with the diagram for $P_{n}(Q)$, and with the diagram in Eq. (17) later, that $T_{2 n}(Q) \cong T_{A_{n}}(Q)$, i.e. we jump two lines on this diagram for every one on the diagram in Eq. (17). Also note that here, unlike $P_{n}(Q)$, there is only a single representation associated with each node. This is because the planar $2 \mathrm{~d}$ lattice allows no possibility of connectivities crossing without themselves becoming connected (i.e. no permutations are allowed).

It is straightforward to compute the exceptional structures in this case (see [2]).

We can summarize the answers to the questions posed at the end of the previous subsection in this case by noting that here the Diagram algebra is isomorphic to the Full algebra.

Perhaps the next two simplest cases to consider are $G=\hat{A}_{n}$ and the daisy graphs (tree graphs consisting of spokes radiating from a central node). They are both extremely illuminating, and we will deal with each in commensurate detail.

\section{The Case $\hat{A}_{n}$}

Here we want to address the following questions:

1. What is the structure of $D_{G}(Q)$ ?

2. What is the structure of $T_{G}(Q)$ ?

3. What is the content of the TM algebra, i.e. the cylindrical Potts model representation?

Everything we do in this section is enthralled by the consequences for $\hat{A}_{n}{ }^{*}$ of the highly useful $A_{n}$ tower relation (15). For $A_{n}$ this is the key to an inductive step

\footnotetext{
* In this paper we choose $\hat{A}_{n}$ to have $n$ vertices rather then $n+1$ (the standard case) to make our notations more convenient
} 
between the structure of the smaller and the larger algebra. Here, of course, we can only manage a weak echo

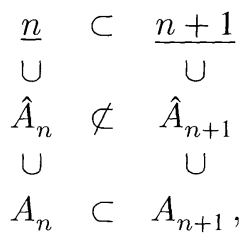

where the vertical inclusions are realized by, for example, omitting $U_{1 n}$ in going from $\hat{A}_{n}$ to $A_{n}$. We are further handicapped by the extra complications of even the simplest cases:

\subsection{The Case $D_{\hat{A}_{n}}(Q)$}

This is the quotient of $T_{\hat{A}_{n}}(Q)$ appropriate for dichromatic polynomials (that is to say, the representations appearing in the TMs for dichromatic polynomials are representations of this algebra [2]), and so it is reasonable to suppose that it plays an important role in the closely related Potts models as well (see Subsect. 4.4), if not in all physical models.

We would like to know the complete structure of the algebra, but physically the key initial question is: What is the generic asymptotic growth rate, with $n$, of dimensions of irreducible representations? The asymptotic growth rate for the $Q$-state Potts representation is $Q$. Clearly if the generic asymptotic growth rate of dimensions of irreducible representations is greater than the actual asymptotic growth rate of the model representation generically containing those irreducibles then eventually the model representation must become smaller than the generic irreducibles and hence a source of non-generic representations. For axiomatic statistical mechanics this crossover number should still be 4 here (i.e. as it is for the open boundary case). This is indeed what we find.

Bearing in mind our tower structure let us begin by recalling the generic Bratelli restriction diagram for the irreducible representations in the ordinary open bounded case, i.e. associated to $T_{A_{n}}(Q) \supset T_{A_{n-1}}(Q)$. Starting with top line $n=0$ we have:

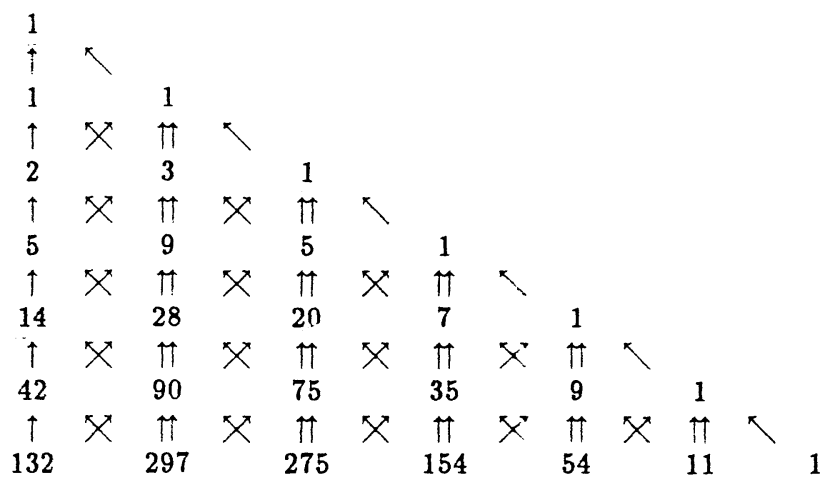

Since the affine case restricts to the open case by omitting $U_{1 n}$ we know that the irreducible representations must decompose as direct sums of the representations above. The irreducible representations may be characterized in essentially the same way as in the open case (quotienting by those permutations of $t=0$ to $t=T$ 
lines discussed above, which in this case are just cyclic permutations, the number of equivalence classes is the same), but the dimensions are different. These may be computed in a number of alternative ways. The above dimensions will be lower bounds. The $P_{n}(Q) / \sim$ dimensions in Sect. 2.6.3 will be upper bounds.

We find the following table of dimensions of irreducibles for $D_{\hat{A}_{n}}(Q)$ [not a restriction diagram this time - see Eq. (16)]:

$\begin{array}{ccccccc}1 & & & & & & \\ 1 & 1 & & & & & \\ 2 & 3 & 1 & & & & \\ 5 & 10 & 6 & 1 & & & \\ 14 & 35 & 28 & 8 & 1 & & \\ 42 & 126 & 120 & 45 & 10 & 1 & \\ 132 & 462 & 495 & 220 & 66 & 12 & 1\end{array}$

and so on. Note that the numbers in column $i$ (counting from $i=0$ on the left) give the dimensions, but correspond in general to more than one inequivalent irreducible, since a semi-direct product with the cyclic group of order $i$ survives from the corresponding product with $\Sigma_{i}$ in the "mean field" case $(G=\underline{n})$. For a general graph $G$ our procedure is first to compute the $P_{n}(Q) / \sim$ type cases (which symmetrise among the possible permutations of distinct connectivities), and then semi-direct product (as in Sect. 2.6.3) with the subgroup of $\Sigma_{\imath}$ consistent with the permutations that can be realised in $G \times \mathbb{Z}$. In this case only cycles are available (and all irreducible dimensions thus multiplied by 1 ).

For example the $S_{3}(2) \Sigma_{ \pm}$basis may be written

$$
\begin{aligned}
& \left\{\left(\left(\left(121^{\prime}\right)\left(32^{\prime}\right)\right) \pm\left(\left(122^{\prime}\right)\left(31^{\prime}\right)\right)\right),\left(\left(\left(232^{\prime}\right)\left(11^{\prime}\right)\right) \pm\left(\left(231^{\prime}\right)\left(12^{\prime}\right)\right)\right)\right. \\
& \quad\left(\left(\left(22^{\prime}\right)\left(131^{\prime}\right)\right) \pm\left(\left(21^{\prime}\right)\left(132^{\prime}\right)\right)\right),\left(\left((1)\left(21^{\prime}\right)\left(32^{\prime}\right)\right) \pm\left((1)\left(22^{\prime}\right)\left(31^{\prime}\right)\right)\right), \\
& \left.\left(\left((2)\left(11^{\prime}\right)\left(32^{\prime}\right)\right) \pm\left((2)\left(12^{\prime}\right)\left(31^{\prime}\right)\right)\right),\left(\left((3)\left(11^{\prime}\right)\left(22^{\prime}\right)\right) \pm\left((3)\left(12^{\prime}\right)\left(21^{\prime}\right)\right)\right)\right\}
\end{aligned}
$$

so the representation of $1_{12}$ for the - case is

$$
\mathscr{R}_{\left(1^{3}\right)}\left(1_{12}\right)=\left(\begin{array}{cccccc}
1 & 0 & 0 & 0 & 0 & 0 \\
0 & 0 & \pm 1 & 0 & 0 & 0 \\
0 & \pm 1 & 0 & 0 & 0 & 0 \\
0 & 0 & 0 & 0 & 1 & 0 \\
0 & 0 & 0 & 1 & 0 & 0 \\
0 & 0 & 0 & 0 & 0 & \pm 1
\end{array}\right)
$$

and is the identity matrix for the + case.

The dimensions may be computed as follows. Let $C_{n, j}$ denote the irreducible representation of $T_{A_{n}}(Q)$ with $j$ distinct lines from $t=0$ to $t=T$; the representation induced from $D_{A_{n}} I_{j} / D_{A_{n}} I_{\jmath-1} D_{A_{n}}$ (i.e. the representation in the $n^{\text {th }}$ row and $j^{\text {th }}$ column of the diagram above, in each case counting from zero on the top, resp. left). Let $\hat{C}_{n, 3}$ denote the corresponding representation of $D_{\hat{A}_{n}}(Q)$. Then

Proposition 12.

$$
\begin{gathered}
\hat{C}_{n, 0} \downarrow_{T_{A_{n}}}=C_{n, 0}, \\
\hat{C}_{n, 1} \downarrow_{T_{A_{n}}}=\sum_{m=0,1,2, \ldots} C_{n, 2 m+1},
\end{gathered}
$$


and for $k=2,3,4, \ldots, n$,

$$
\hat{C}_{n, k} \downarrow_{T_{A_{n}}}=\sum_{m=0,1,2, \ldots, n-k} C_{n, k+m} .
$$

Proof. The dimension $C_{n, 0}$ is given by the number of elements of $S_{n}=S_{n}(0)$ such that $i \sim j, k \sim l$, and $i<k<j$ implies that $i<l<j$ [2]. The first equation comes from noting that the same constraint applies for $\hat{C}_{n, 0}$.

The dimension $C_{n, 1}$ is given by the number of elements of $S_{n}(1)$ such that $i \sim j$, $k \sim 1$, and $i<k<j$ implies that $i<l<j$; and such that $i \sim j$ implies no primed part contains $k$ such that $i<k<j$. The second equation comes from noting that the difference between this and $\hat{C}_{n, 1}$ is elements in the basis for the latter where a connection $i \sim j$ does not prevent $k$ such that $i<k<j$ appearing in a primed part (we write $k \sim \infty$ ). Consider these elements and modify them by cutting the line from $i$ to $j$ and attaching each loose end separately to $\infty$ (i.e. put these two in separate primed parts). That this is the correct move follows from the action of the generators of $T_{A_{n}}(Q)$ on the basis states of $\hat{C}_{n, 1}$. These elements include the whole of $C_{n, 3}$ plus any for which there was originally a further $i_{2} \sim j_{2}$ and intermediate $k_{2} \sim \infty$. Cutting again, and so on, we get the required result.

For the third equation, note that $\hat{C}_{n, k} \supset C_{n, k}$. The difference is elements where a line to $\infty$ is "isolated" from $\infty$ in $C_{n, k}$ as above. The innermost of these isolating lines may have its own line to $\infty$ also (subsequent ones may not, even in $\hat{C}_{n, k}$ ). Thus we get either zero or one line to $\infty$ becoming two lines to $\infty$ by cutting at the problem point. The new element is either in $C_{n, k+1}$ or $C_{n, k+2}$ or still illegal (but now with either $k+1$ or $k+2$ lines to $\infty$ ). It is straightforward to check that all of these two bases is generated in this way. Cutting again on the illegal elements we may iterate to obtain the required results. QED.

From Proposition 12 the dimensions of representations in $A$ and $\hat{A}$ cases are linearly related. Therefore the asymptotic growth rate is equal to 4 in both cases.

\subsection{The Full TL Case $T_{\hat{A}_{n}}(Q)$ : Explicit Example for $n=2$}

4.2.1. Generalities. Consider the unital associative algebra over $\mathbb{C}$ defined by generators

$$
1, U_{1}, U_{2,}, U_{12}, U_{21}
$$

and relations $1-4$.

This is a "degenerate" case of $T_{G}(Q)$, i.e. $G=\hat{A}_{2}$ (from the diagrammatic point of view we have two bonds between 1 and 2 , so $U_{12} \neq U_{21}$ ). However, it is the simplest interesting case to consider.

It is useful to get an idea of the problem by simply beginning to list linearly independent words in this algebra by length:

$$
\begin{gathered}
1, \\
U_{1 .}, U_{2 .}, U_{12}, U_{21}, \\
U_{1 .} U_{2 .}, U_{1 .} U_{12}, U_{1 .} U_{21}, U_{21} U_{12}, U_{2} U_{12}, U_{2 .} U_{21}, U_{12} U_{1 .}, U_{12} U_{2 .}, U_{21} U_{1 .}, U_{21} U_{2 .}, \\
U_{1 .} U_{2 .} U_{12}, U_{1 .} U_{12} U_{2 .}, U_{1 .} U_{21} U_{2 .}, U_{1 .} U_{21} U_{12}, U_{2 .} U_{12} U_{1 .}, U_{1} U_{2 .} U_{21}, \\
U_{12} U_{1 .} U_{2 .}, U_{2 .} U_{12} U_{21}, U_{21} U_{1 .} U_{2 .}, U_{21} U_{2 .} U_{12}, U_{21} U_{1 .} U_{12}, U_{12} U_{1} U_{21}
\end{gathered}
$$


$\ldots$ and so on (we have given the complete list up to length 3). Clearly we need to be able to get some control of this explosion of elements. Our treatment of $P_{n}(Q)$ tells us how to do this. There we were able to filter the algebra through a sequence of double sided ideals generated by certain idempotents. Since that was a quotient algebra of this, we still have these elements as candidates for primitive idempotents. Let us look at the left sided ideal generated by (unnormalized) idempotent $U_{1} U_{2}$. Organising the words by length we have the following structure

$$
\begin{array}{ccc}
U_{12} U_{1 .} U_{2 .} & U_{1 .} U_{2 .} & \\
\ldots & U_{12} U_{21} U_{1 .} U_{2 .} & U_{21} U_{1 .} U_{2 .} \\
U_{1 .}\left(U_{12} U_{21} U_{1 .} U_{2 .}\right)^{k} & \left(U_{12} U_{21} U_{1 .} U_{2 .}\right)^{k} & \cdots \\
U_{12} U_{1 .} U_{2 .}\left(U_{12} U_{21} U_{1 .} U_{2 .}\right)^{k} & U_{1 .} U_{2 .}\left(U_{12} U_{21} U_{1 .} U_{2 .}\right)^{k} & U_{2 .}\left(U_{12} U_{21} U_{1 .} U_{2 .}\right)^{k} \\
U_{21} U_{1 .} U_{2 .}\left(U_{12} U_{21} U_{1 .} U_{2 .}\right)^{k}
\end{array}
$$

The ideal generated by $U_{1 .} U_{2 .}\left(U_{12} U_{21} U_{1} U_{2 .}\right)^{k}$ (any natural number $k$ ) is a proper invariant subspace (see Proposition 13). There is an injective map from $T U_{1} U_{2}$. into $T U_{1} U_{2 .}\left(U_{12} U_{21} U_{1 .} U_{2 .}\right)^{k}$, so the full ideal $T U_{1} U_{2}$ is isomorphic to a direct product of the $\dot{k}=0$ sector with the natural numbers under addition, $(\mathbb{N},+)-$ which is abelian. A general source of irreducible representations is thus the quotient of $T U_{1} U_{2}$. by

$$
U_{1 .} U_{2 .}\left(U_{12} U_{21} U_{1 .} U_{2 .}\right)=\alpha U_{1 .} U_{2 .}
$$

where $\alpha \in \mathbb{C}$ corresponds to the irrep. of $(\mathbb{N},+)$ given by $R(1)=\alpha$.

These quotients have a symmetric inner product \langle\rangle defined by

$$
A B^{T}=\langle A B\rangle U_{1} U_{2 .},
$$

with Gram matrix, in the basis

$$
\begin{aligned}
& \left\{U_{1 .} U_{2 .}, U_{12} U_{1 .} U_{2 .}, U_{21} U_{1 .} U_{2 .}, U_{12} U_{21} U_{1 .} U_{2 .},\right. \\
& \left.U_{1 .} U_{12} U_{21} U_{1 .} U_{2 .}, U_{2 .} U_{12} U_{21} U_{1 .} U_{2 .}\right\}
\end{aligned}
$$

that reads

$$
\mathrm{Gr}_{n=2, \imath=0}=\left(\begin{array}{cccccc}
Q & \sqrt{Q} & \sqrt{Q} & \alpha & \alpha \sqrt{Q} & \alpha \sqrt{Q} \\
\sqrt{Q} & Q & \alpha & \alpha \sqrt{Q} & \alpha & \alpha \\
\sqrt{Q} & \alpha & Q & \alpha \sqrt{Q} & \alpha & \alpha \\
\alpha & \alpha \sqrt{Q} & \alpha \sqrt{Q} & \alpha Q & \alpha \sqrt{Q} & \alpha \sqrt{Q} \\
\alpha \sqrt{Q} & \alpha & \alpha & \alpha \sqrt{Q} & \alpha Q & \alpha^{2} \\
\alpha \sqrt{Q} & \alpha & \alpha & \alpha \sqrt{Q} & \alpha^{2} & \alpha Q
\end{array}\right)
$$

The latter has for determinant

$$
\operatorname{det} \mathrm{Gr}_{2,0}=\alpha^{3}(\alpha-Q)^{4}\left((Q-2)^{2}-\alpha\right) \text {. }
$$

With the relation (22) $U_{1} U_{2}$. $/ Q$ becomes a primitive idempotent, so the representation is indecomposable. Taking also into account the Gram matrix it is thus generically irreducible. 




Fig. 7. Boundary diagram for $U_{1} U_{2}$. in $D_{\hat{A}_{2}}(Q)$ drawn on a cylinder

We still have a representation in terms of boundary diagrams but now lines are non-contractible non-overlapping segments on, and beginning and ending on the boundaries of, the twice punctured sphere without any seam: Fig. 7. It follows from the structure in (21) that this is a faithful representation. The ideal $T U_{1} U_{2}$ is infinite dimensional. We can write down a sequence of quotients which exhibit the invariant subspaces indicated by the determinant (25). With the above topological picture in mind, the first trial quotient with which we might hope to collapse the ideal into a non-trivial finite dimensional one, is of the form

$$
\left(e 1+a U_{12}+b U_{21}\right) U_{1} U_{2 .}=0
$$

(e, $a, b$ scalars) but then

$$
U_{1 .}\left(e 1+a U_{12}+b U_{21}\right) U_{1 .} U_{2 .}=(\sqrt{Q} e+a+b) U_{1 .} U_{2 .}=0
$$

and

$$
U_{12} U_{21}\left(e 1+a U_{12}+b U_{21}\right) U_{1 .} U_{2 .}=(e+\sqrt{Q}(a+b)) U_{12} U_{21} U_{1} U_{2 .}=0
$$

so either $Q=1$ (an exceptional case, which we will discount for the moment), or $e=0$ and $a=-b$ (= $=-1$, without loss of generality). Diagramatically we have equivalence between the pictures of Fig. 8 and Fig. 9, i.e. we have legalized this

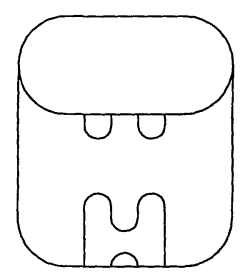

Fig. 8. Boundary diagram for $U_{1} U_{2} U_{12}$ in $D_{\hat{A}_{2}}(Q)$

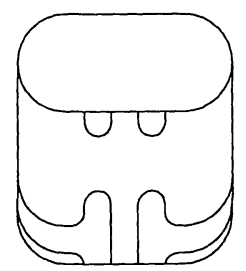

Fig. 9. Boundary diagram for $U_{1} U_{2} \cdot U_{21}$ in $D_{\hat{A}_{2}}(Q)$ 


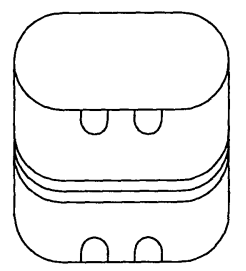

Fig. 10. Boundary diagram for $p_{1}=2$ in $D_{\hat{A}_{2}(Q)}$

topologically non-trivial move. Altogether the prospective quotient ideal is spanned by

$$
\left\{U_{1 .} U_{2 .}, U_{12} U_{1 .} U_{2 .}\right\}
$$

and $U_{1} U_{2}$ is a primitive idempotent (so the ideal is indecomposable, and generically simple). It is easy to see that this module is isomorphic to the 2 dimensional representation of the diagram algebra, which is, in turn, the one induced by braid translation

$$
U_{1 n}=\left(\prod_{i=1}^{n-1} g_{\imath} g_{\imath \imath+1}\right)^{-1} U_{1}\left(\prod_{i=1}^{n-1} g_{i .} g_{i i+1}\right)
$$

( $g=1-q^{ \pm 1} U$, where $q+q^{-1}=\sqrt{Q}$ ) from the $A_{n}$ case (see Fig. 4.2.1).

There are many other possible quotients which we may try. For example,

$$
U_{12} U_{21} U_{1} U_{2 .}=k U_{1} U_{2 .},
$$

where $k$ is a scalar. In this case

$$
U_{21} U_{12} U_{12} U_{21} U_{1} U_{2 .}=Q U_{21} U_{12} U_{1 .} U_{2 .}=U_{21} U_{12} k U_{1 .} U_{2}
$$

so $k=Q$, and

$$
U_{1 .} U_{21} U_{12} U_{21} U_{1 .} U_{2 .}=U_{1}, U_{21} k U_{1 .} U_{2 .}=k U_{1 .} U_{2 .}=Q k U_{1 .} U_{2}
$$

so $Q=1$. For the moment we will disregard such exceptional $Q$ cases. As is probably already apparent, the way to get generic cases is to make quotients corresponding to introducing topological moves [as Eq. (26)]. There are various options corresponding to simply dragging a line across a puncture, which we will leave to the interested reader. Those which give rise to representations in which a new parameter actually survives unconstrained are characterized in the next section:

4.2.2. Free-Non-Contractible Loops. By reference to the boundary diagram picture we note that a natural class of (finite dimensional) quotients to consider is

$$
U_{1 .} U_{2 .}\left(U_{12} U_{21} U_{1 .} U_{2 .}\right)^{p_{1}}=\alpha\left(p_{1}, p_{2}\right) U_{1 .} U_{2 .}\left(U_{12} U_{21} U_{1 .} U_{2 .}\right)^{p_{2}}
$$

This corresponds diagrammatically to establishing a rule in which $2 p_{1}$ free noncontractible loops (see Fig. 10) may be replaced by $2 p_{2}<2 p_{1}$ loops at cost of an overall factor $\alpha\left(p_{1}, p_{2}\right)$. The dimension is $6 p_{1}$. However the representation implied by (27) reduces to a direct sum of cases $p_{1}=1, p_{2}=0$ (22) for various choices of $\alpha$. Consider for instance the case $p_{2}=0$. Call $X$ any element of the basis (24). The 
subspace spanned by ("topological" Fourier transform)

$$
|X, k\rangle=\sum_{p=0}^{p_{1}-1} \alpha\left(p_{1}\right)^{\frac{p k}{p_{1}}} X\left(U_{12} U_{21} U_{1 .} U_{2 .}\right)^{p}
$$

is invariant for each $k=0, \ldots, p_{1}-1$. The relation (22) holds with $\alpha=\alpha\left(p_{1}\right)^{\frac{k}{p_{1}}}$. If $p_{2} \neq 0$ the sector with $2 p_{2}$ loops is an invariant subspace isomorphic to the case $p_{2}=0$. The quotient has $\alpha=0$.

Thus the only case to consider is the aforementioned (22),

$$
U_{1 .} U_{2 .} U_{12} U_{21} U_{1 .} U_{2 .}=\alpha U_{1} U_{2}
$$

with $\alpha$ a scalar. A basis for the full ideal with the above quotient is given by (24), i.e. this produces a 6 dimensional algebra (without unit). Here $U_{1} U_{2} / Q$ is a primitive idempotent, so the corresponding representation is indecomposable. In our basis we have (with all omitted entries zero)

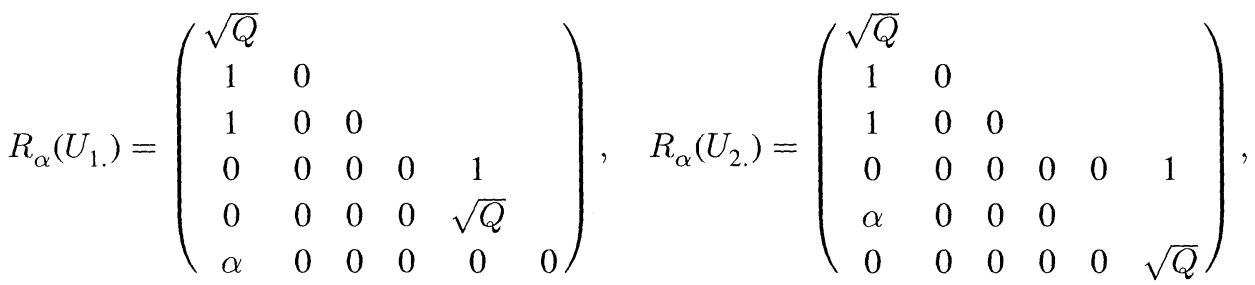

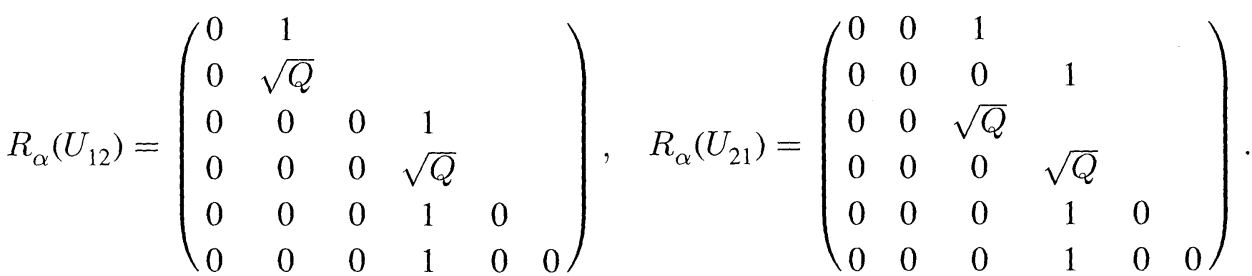

Proposition 13. The algebra $T_{\hat{A}_{2}}(Q)$ is infinite dimensional.

Proof. We prove that the above set of representations characterized by the indeterminate $\alpha$ are mutually inequivalent. This follows by direct computation of

$$
\operatorname{trace}\left(R_{\alpha}\left(U_{12} U_{21} U_{1} U_{2 .}\right)\right)=\alpha . \quad \text { QED }
$$

A straightforward generalisation of this proof serves to show that the $\hat{A}_{n}$ algebra is infinite dimensional for all $n>1$.

If we want to examine the exceptional structure of the present set of representations with respect to $a$ then one suitable basis is

$$
\begin{aligned}
& \left\{U_{1 .} U_{2 .},\left(U_{12}+U_{21}\right) U_{1 .} U_{2 .},\left(U_{12}-U_{21}\right) U_{1 .} U_{2 .},\left(\sqrt{Q} U_{12}-U_{12} U_{21}\right) U_{1 .} U_{2 .}\right. \\
& \left.\left(\sqrt{Q}-U_{1 .} U_{12} U_{21}\right) U_{1 .} U_{2 .},\left(\sqrt{Q}-U_{2 .} U_{12} U_{21}\right) U_{1 .} U_{2 .}\right\}
\end{aligned}
$$


in terms of which we have

$$
\begin{aligned}
& U_{1 .}=\left(\begin{array}{cccccc}
\sqrt{Q} & & & & & \\
2 & 0 & & & & \\
0 & 0 & 0 & & & \\
0 & 0 & 0 & 0 & 1 & \\
0 & 0 & 0 & 0 & \sqrt{Q} & \\
Q-\alpha & 0 & 0 & 0 & 0 & 0
\end{array}\right), \quad U_{2 .}=\left(\begin{array}{cccccc}
\sqrt{Q} & & & & & \\
2 & 0 & & & & \\
0 & 0 & 0 & & & \\
0 & 0 & 0 & 0 & 0 & 1 \\
Q-\alpha & 0 & 0 & 0 & & \\
0 & 0 & 0 & 0 & 0 & \sqrt{Q}
\end{array}\right), \\
& U_{12}=\left(\begin{array}{cccccc}
0 & 1 / 2 & 1 / 2 & & & \\
0 & \sqrt{Q} & \sqrt{Q} & -1 & \\
0 & 0 & 0 & 1 & \\
0 & 0 & 0 & \sqrt{Q} & \\
0 & 0 & 0 & 1 & 0 & \\
0 & 0 & 0 & 1 & 0 & 0
\end{array}\right), \quad U_{21}=\left(\begin{array}{cccccc}
0 & 1 / 2 & -1 / 2 & & \\
0 & \sqrt{Q} & 0 & -1 & \\
0 & 0 & \sqrt{Q} & 1 & \\
0 & 0 & 0 & 0 & \\
0 & 0 & -\sqrt{Q} & 1 & 0 \\
0 & 0 & -\sqrt{Q} & 1 & 0 & 0
\end{array}\right) .
\end{aligned}
$$

We note that when $\alpha=Q$ (cf. the Gram matrix) there is a two dimensional invariant subspace corresponding to the quotient

$$
U_{12} U_{1 .} U_{2 .}=U_{21} U_{1 .} U_{2 .},
$$

which we have already identified with the diagram representation [Eq. (26)].

Note that the left sided ideal generated by $U_{12} U_{21}$ will be "dual" to this one (i.e. isomorphic up to the usual duality transformation).

4.2.3. The Case $T U_{1} / T I_{0} T$. The word structure of the left sided ideal generated by $U_{1}$. (quotienting by $T U_{1} U_{2} T$ ) is

$$
\begin{aligned}
& U_{1} \text {. } \\
& U_{12} U_{1} \\
& U_{2 .} U_{12} U_{1} . \quad U_{12} U_{21} U_{1} . \quad U_{2 .} U_{21} U_{1} \\
& U_{21} U_{2} U_{12} U_{1} \text {. } \quad U_{1} U_{12} U_{21} U_{1} \text {. } \quad U_{2} U_{12} U_{21} U_{1} . \quad U_{12} U_{2} U_{21} U_{1} \text {. }
\end{aligned}
$$

and so on. By the same argument as before, the appropriate quotient here is

$$
U_{1 .} U_{21} U_{2} U_{12} U_{1 .}=\alpha_{1} U_{1}
$$

and

$$
U_{1 .} U_{12} U_{21} U_{1 .}=z U_{1}
$$

Notice that (28) implies

$$
U_{1 .} U_{12} U_{2 .} U_{21} U_{1 .}=\alpha_{1}^{-1} U_{1} .
$$

The only consistent choices are $z=0$ or $z=\sqrt{Q}, \alpha_{1}=1$. This then reduces to a four dimensional ideal. Once again there is a symmetric inner product, with Gram matrix in the basis $\left\{U_{1}, U_{1} U_{12}, U_{1} U_{12} U_{2}, U_{1} U_{12} U_{2 .} U_{21}\right\}$,

$$
\mathrm{Gr}_{2,1}=\left(\begin{array}{cccc}
\sqrt{Q} & 1 & 0 & \alpha_{1} \\
1 & \sqrt{Q} & 1 & z \\
0 & 1 & \sqrt{Q} & 1 \\
\alpha_{1}^{-1} & z & 1 & \sqrt{Q}
\end{array}\right)
$$




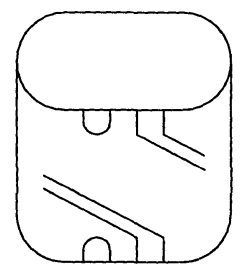

Fig. 11. Boundary diagram for $U_{1} U_{12} U_{2} U_{21} U_{1}$

with determinant

$$
\left(-\left(\alpha_{1}+\alpha_{1}^{-1}\right)+Q^{2}-4 Q+2\right)
$$

in case $z=0$. In case $z=\sqrt{Q}$ the irreducible quotient is isomorphic to $\hat{C}_{n .1}$.

In order to see what is going on it is useful to look again at Fig. 11: a boundary gets a weight $\alpha_{1}^{ \pm 1}$ when going around the cylinder.

\subsection{The Full Case $T_{\hat{A}_{n}}(Q)$}

To get further insight we shall use the analysis of the so called 6-vertex model carried out in [9]. Introduce the space $\mathbb{C}^{4 n}$ of dimension $2^{2 n}$ considered as tensor product of $2 n$ fundamental representations of the quantum algebra $U_{q} s l(2)$ (with generators $\left.X_{ \pm}, H\right)$. Set as usual $\sqrt{Q}=q+q^{-1}$ and consider the matrix representation of $T_{\hat{A}_{n}}(Q)$, where $U_{\imath}\left(U_{i, \imath+1}\right)$ acts as identity everywhere except in the $(2 i-1)^{\text {th }}$ and $(2 i)^{\text {th }}\left[(2 i)^{\text {th }}\right.$ and $\left.(2 i+1)^{\text {th }}\right]$ copies of $\mathbb{C}^{2}$ where it acts as the $4 \times 4$ matrix,

$$
U=\left(\begin{array}{cccc}
0 & 0 & 0 & 0 \\
0 & q^{-1} & -1 & 0 \\
0 & -1 & q & 0 \\
0 & 0 & 0 & 0
\end{array}\right)
$$

while the special boundary element $U_{1 n}$ acts between the first and last copies of $\mathbb{C}^{2}$ as

$$
U=\left(\begin{array}{cccc}
0 & 0 & 0 & 0 \\
0 & q^{-1} & -x & 0 \\
0 & -x^{-1} & q & 0 \\
0 & 0 & 0 & 0
\end{array}\right)
$$

where $x$ is a complex parameter. For each value of the total weight $h=0, \pm 1, \ldots, \pm n$ this provides a representation of $T_{\hat{A}_{n}}(Q)$ with continuous parameter $x$. We shall refer to this representation as $R_{n}\left(\begin{array}{l}x \\ h\end{array}\right)$. It has for dimension the binomial coefficient $C_{2 n}^{n-h}$. The cases $\pm h$ are isomorphic and we restrict without loss of generality to the study of $h \geq 0$.

4.3.1. The Case $T I_{0}$. The same analysis as $n=2$ works for any $n$, i.e. all irreducible representations may be found by putting

$$
\left(U_{1}, U_{2} \ldots U_{n}\right) U_{12} U_{23} \ldots U_{n 1}\left(U_{1} U_{2} \ldots U_{n .}\right)=\alpha\left(U_{1}, U_{2} \ldots U_{n .}\right) \text {. }
$$

Proposition 14. The dimension of this sector is $\mathscr{C}_{n}=(n+1) \cdot C_{n, 0}=C_{2 n}^{n}$. 
This is because in addition to the $C_{n, 0}$ basic connectivities in the $i=0$ (zero propagating connections) sector we get a new basis state from each one of these by picking any of the $n$ sites and moving it round a closed non-contractible path.

The idempotent $I_{0}$ is primitive with the quotient (32) so $T I_{0}$ modulo (32) is indecomposable. It has a natural basis of words in the generators generalizing (24). The representation induced from this basis we will call $\mathscr{T}_{n}\left(\begin{array}{l}x \\ 0\end{array}\right)$.

There is a simple intertwiner between $\mathscr{T}_{n}\left(\begin{array}{l}x \\ 0\end{array}\right)$ and the vertex model $R_{n}\left(\begin{array}{l}x \\ 0\end{array}\right)$, where

$$
\alpha=\left(x^{1 / 2}+x^{-1 / 2}\right)^{2} .
$$

Associate with each boundary diagram a state in the $h=0$ sector of the vertex model basis by giving all possible orientations to boundaries with the weights

$$
\uparrow=\lambda \psi=q^{-1 / 2}, \quad \Psi \uparrow=\psi \lambda=q^{1 / 2}
$$

and

$$
\begin{aligned}
& \Psi \ldots \uparrow=q^{-1 / 2} x^{1 / 2}, \quad \downarrow \ldots\left\lceil=q^{-1 / 2} x^{-1 / 2},\right. \\
& \uparrow \ldots \Psi=-q^{1 / 2} x^{-1 / 2}, \quad \nmid \ldots \downarrow=-q^{1 / 2} x^{1 / 2},
\end{aligned}
$$

where up (down) arrow indicates $h= \pm 1 / 2$. This is best illustrated by an example. To $U_{12} U_{21} U_{1} U_{2}$. for $n=2$ is associated for instance

$$
\begin{aligned}
& -q^{1 / 2} x^{-1 / 2}|+\rangle \otimes\left(q^{-1 / 2}|+-\rangle-q^{1 / 2}|-+\rangle\right) \otimes|-\rangle, \\
& +q^{-1 / 2} x^{1 / 2}|-\rangle \otimes\left(q^{-1 / 2}|+-\rangle-q^{1 / 2}|-+\rangle\right) \otimes|+\rangle .
\end{aligned}
$$

The idempotent $U_{1} \ldots U_{n} / Q^{n / 2}$ projects the sector $h=0$ of the vertex model onto a one dimensional subspace. The intertwiner is then obtained by acting on this subspace with the representations $R_{n}\left(\begin{array}{l}x \\ 0\end{array}\right)$. This intertwiner is $x$-generically invertible as can be shown by noticing that in every row and column there is exactly one occurrence of the highest power of $q$ (or $q^{-1}$ ).

The use of the vertex model is to determine a minimal set of invariant subspaces over the non-generic values of $x$. We will then saturate the bound by reference to $\mathrm{Gr}_{n, 0}$. It is not convenient to work entirely in $\mathscr{T}_{n}$ as the $U_{q} s l(2)$ action is not manifest there.

Commutation with $U_{q} s l(2)$ is not possible in $\hat{A}_{n}$ due to boundary effects, but for non-generic values of the parameter $x, x=q^{ \pm 2 k}$ ( $k$ positive integer) there is a commutative diagram as the $h=0$ case of

$$
\begin{gathered}
R_{n}\left(\begin{array}{c}
x=q^{2 k} \\
h
\end{array}\right) \stackrel{X_{+}^{k-h}}{\longrightarrow} R_{n}\left(\begin{array}{c}
x=q^{2 h} \\
k
\end{array}\right) \\
\downarrow \\
R_{n}\left(\begin{array}{c}
x=q^{2 k} \\
h
\end{array}\right) \stackrel{X_{+}^{k-h}}{\longrightarrow} R_{n}\left(\begin{array}{c}
x=q^{2 h} \\
k
\end{array}\right)
\end{gathered}
$$


$(k>h)$ where $X_{+}$is the raising operator in $U_{q} \operatorname{sl}(2)$ [9]. This allows us to identify an invariant subspace $\varrho_{n}\left(\begin{array}{l}k \\ 0\end{array}\right)=\operatorname{Ker} X_{+}^{k}$. A similar result holds by replacing $q$ with $q^{-1}$. Its dimension is $C_{2 n}^{n}-C_{2 n}^{n-k}$. We note that the intertwiner is singular at these values.

In addition to this, another case that was overlooked in [9] is $\alpha=0, x=-1$. Using the symmetry of the TL algebra under $q \rightarrow-q$ one can establish in that case the existence of a long commutative diagram where the morphisms are given by generators of $U_{i} s l(2)$. This implies that there is an invariant subspace $\varrho_{n}^{\prime}$ of dimension $C_{2 n}^{n}-C_{2 n}^{n-1}+C_{2 n}^{n-2}=\ldots=\frac{1}{2} C_{2 n}^{n}$.

Proposition 15. The determinant of the Gram matrix has a factor $\alpha^{C_{n} / 2}$ and is overall order $2^{2 n-1}$ in $\alpha$.

Proof. The factor arises since half the basis states begin with one non-contractible loop (and $A B^{T}$ has an even number). An upper bound on the number of closed loops formed by the $A B^{T}$ contribution to a single determinant factor (23) is the total number of occurrences of $U_{1 n}$ in the $T I_{0}$ basis (since we cannot build a loop without the periodic closure). In the $T I_{0}$ basis there are $C_{2 n}^{2 w-1+n}-C_{2 n}^{2 w+1+n}$ words with $w$ factors of $U_{1 n}$, but

$$
\sum_{w=1,2 \ldots} w\left(C_{2 n}^{2 w-1+n}-C_{2 n}^{2 w+1+n}\right)=2^{2 n-1} .
$$

On the other hand the complement of an invariant subspace contributes a zero of order its dimension to detGr. The sum of dimensions of the complements of the invariant subspaces we have so far identified in $R_{n}$ is

$$
\sum_{i=1}^{n} C_{2 n}^{n-1}+\frac{1}{2} C_{2 n}^{n}=\frac{1}{2} 2^{2 n}
$$

where we used

$$
(1+1)^{2 n}=\sum_{i=0}^{2 n} C_{2 n}^{i}=C_{2 n}^{n}+2 \sum_{i=1}^{n} C_{2 n}^{n-i} .
$$

Therefore the bound is already saturated and this determinant is

$$
\operatorname{det} \mathrm{Gr}_{n, 0} \propto \alpha^{\frac{1}{2}} C_{2 n}^{n} \prod_{\imath=1}^{n}\left(\alpha-P_{i}^{2}\right)^{C_{2 n}^{n-\imath}}
$$

where we introduced

$$
P_{k}=q^{k}+q^{-k} .
$$

The $P_{k}$ are polynomials in $\sqrt{Q}$ determined by the induction

$$
P_{k}=\sqrt{Q} P_{k-1}-P_{k-2}, P_{0}=2, P_{1}=\sqrt{Q} .
$$

We have therefor proven

Proposition 16. The representation $R_{n}\left(\begin{array}{l}x \\ 0\end{array}\right)$ has dimension $C_{2 n}^{n}$ and is irreducible except at $x=q^{ \pm 2 k}, k=1, \ldots, n$, and $x=-1$. When $x=q^{ \pm 2 k}$ it contains $\varrho_{n}\left(\begin{array}{c}k \\ 0\end{array}\right)$ 
with dimension $C_{2 n}^{n}-C_{2 n}^{n-k}$ as an irreducible component. When $x=-1$ it contains $\varrho_{n}^{\prime}$ with dimension $\frac{1}{2} C_{2 n}^{n}$.

It is easy to show that the particular $\varrho_{n}\left(\begin{array}{l}1 \\ 0\end{array}\right)$ representation is isomorphic with the one induced from $C_{n, 0}$ by braid translation.

4.3.2. The Case $T I_{h} / T I_{h-1} T$. All irreducible representations may be found by introducing the quotient relation constructed as follows. Take the word $I_{h}$ (here $\left.I_{h}=\prod_{i=1}^{n-h}\left(U_{i .} / \sqrt{Q}\right)\right)$ and rotate the top once around the cylinder clockwise holding the bottom fixed. Equate this new word with $\alpha_{h} I_{h}$. The same result with $\alpha_{h}^{-1}$ holds then for counterclockwise rotation. For instance if $h=1$,

$$
\begin{aligned}
& \left(U_{1 .} \ldots U_{(n-1) .}\right) U_{n 1}\left(U_{2 .} \ldots U_{n .}\right) U_{12} \ldots U_{n-1, n}\left(U_{1 .} \ldots U_{(n-1) .}\right) \\
& \quad=\alpha_{1}\left(U_{1} \ldots U_{(n-1) .}\right), \\
& \left(U_{1 .} \ldots U_{(n-1) .}\right) U_{12} \ldots U_{n-1, n}\left(U_{2 .} \ldots U_{n .}\right) U_{n 1}\left(U_{1 .} \ldots U_{(n-1)}\right) \\
& \quad=\alpha_{1}^{-1}\left(U_{1 .} \ldots U_{(n-1) .}\right) .
\end{aligned}
$$

In the $h=1$ case this does not give a finite dimensional representation. We need the additional relation

$$
\left(U_{1} . U_{2 .} \ldots U_{(n-1) .}\right) U_{12} U_{23} \ldots U_{n 1}\left(U_{1 .} U_{2 .} \ldots U_{(n-1) .}\right)=z\left(U_{1 .} U_{2 .} \ldots U_{(n-1) .}\right) .
$$

This is because a single connectivity can pass through a non-contractible closed loop, while multiple connectivities cannot. As in the $n=2$ case, either $z=\sqrt{Q}, \alpha_{1}=1$ in which case this is just $\hat{C}_{n, 1}$, or $z=0$ and $\alpha_{1}$ is unconstrained. Putting $z=0$ brings $h=1$ into line with other $h>0$ for which this issue does not arise.

The left sided ideal $T I_{h} / T I_{h-1} T$ we call $T[h]$. Quotienting further by (37) [and by (38) with $z=0$ in the case $h=1]$ we obtain $\mathscr{T}_{n}\left(\begin{array}{c}\alpha_{h} \\ h\end{array}\right)$.

Proposition 17. The dimension of $\mathscr{T}_{n}\left(\begin{array}{c}\alpha_{h} \\ h\end{array}\right)$ is $C_{2 n}^{n-h}$.

Proof. Recall that this number is the number of walks of length $2 n$ on the Pascal triangle from the beginning to height $2 h$. We associate $U_{1} \ldots U_{(n-h)}$. to the lowest such walk that does not visit negative heights. The rest of the correspondence is obtained by adding diamonds to represent each $U$ until we reach the same walk again via spacial periodicity and relation (37). Note that any periodic rectangle defines a different but isomorphic basis by the relation (37).

The intertwiner given in the case $T I_{0} T$ is also an intertwiner between $\mathscr{T}_{n}\left(\begin{array}{c}\alpha_{h} \\ h\end{array}\right)$ and $R_{n}\left(\begin{array}{l}x \\ h\end{array}\right)$, where $\alpha_{h}=x^{h}$.

The commutative diagram (35) holds as well here, allowing us to identify a sequence of invariant subspaces $\varrho_{n}\left(\begin{array}{l}k \\ h\end{array}\right)$ of dimension $C_{2 n}^{n-h}-C_{2 n}^{n-k}, h<k \leq n$. By a similar argument to the case $h=0$ we can find an upper bound on the $x$-order of the Gram matrix $\sum_{k=h+1}^{n} C_{2 n}^{n-k}$. The above sequence of invariant subspaces provides 
enough power of $\alpha_{h}$ to saturate this bound. The determinant of the Gram matrix reads therefore

$$
\operatorname{det} \mathrm{Gr}_{n, h}= \pm \prod_{i=h+1}^{n}\left(-\left(x+x^{-1}\right)+P_{2 i}\right)^{C_{2 n}^{n-\imath}}
$$

and

Proposition 18. The representation $R_{n}\left(\begin{array}{l}x \\ h\end{array}\right)$ has dimension $C_{2 n}^{n-h}$ and is irreducible except at $x=q^{ \pm 2 k}, k=h+1, \ldots, n$. When $x=q^{ \pm 2 k}$ it contains $\varrho_{n}\left(\begin{array}{l}k \\ h\end{array}\right)$ with dimension $C_{2 n}^{n-h}-C_{2 n}^{n-k}$ as an irreducible component.

The representation $\varrho_{n}\left(\begin{array}{c}h+1 \\ h\end{array}\right)$ is the one isomorphic to the representation induced from $C_{n, h}$ by braid translation.

\subsection{The Potts model}

As an application we now determine which of the above irreducible representations appear in the toroidal Potts model. Consider a most general inhomogeneous $Q$ state Potts model on $\hat{A}_{n} \times \mathbb{Z}$ with periodic boundary conditions in time direction. Couplings can vary from edge to edge, corresponding to inhomogeneous edge transfer matrices of the form given in the introduction. We restrict for simplicity to $Q \in[0,4]$ and for $Q$ not an integer define the Potts model partition function by the usual dichromatic polynomial. Introduce also the partition function $Z_{h, h^{\prime}}$ of the associated 6 -vertex model with periodic boundary conditions and such that the total $H$ number encountered along the space (resp. time) direction is equal to $h\left(h^{\prime}\right)$. Consider the same model with twisted boundary conditions in space direction, as given in [9]. Then the trace of the transfer matrix in the representations $R_{n}\left(\begin{array}{l}x \\ h\end{array}\right)$ reads

$$
Z\left(\begin{array}{l}
x \\
h
\end{array}\right)=\sum_{h^{\prime}} x^{h^{\prime}} Z_{h, h^{\prime}}
$$

In this formula and in what follows we do not take into account the truncation of various sums due to the finite size of the system, which can be easily reinstalled. Let us set

$$
q=\exp \left(\frac{i \pi}{\mathscr{N}^{*}}\right)
$$

where $\mathscr{N}$ is a real number. It will turn out that we can restrict to the case where $x$ is of modulus one, which we can always parametrize as $x=q^{2 t}, t \in[0, \mathscr{N}]$. Then (40) can be inverted to give

$$
Z_{h, h^{\prime}}=\frac{1}{\mathscr{N}} \int_{0}^{\mathscr{N}} Z\left(\begin{array}{c}
x=q^{2 t} \\
h
\end{array}\right) e^{-2 i \pi t h^{\prime} / \mathscr{N}} d t
$$

The partition function of the $Q$ state Potts model was then rewritten in [22] as

$$
Z=Z^{(1)}+Z^{(2)}
$$


with

$$
Z^{(1)}=\sum_{h, h^{\prime}} Z_{h, h^{\prime}} \cos \left[\frac{2 \pi}{\mathscr{N}}\left(h \wedge h^{\prime}\right)\right]
$$

and

$$
Z^{(2)}=\frac{Q-1}{2} \sum_{h, h^{\prime}} Z_{h, h^{\prime}} \cos \left[\pi\left(h \wedge h^{\prime}\right)\right]
$$

where $\wedge$ denotes the greatest common divisor. This was established by analysis of the weights of non-contractible loops (in space and time directions) in the vertexboundaries correpondence. To get the desired result we therefore have to plug in these formulas the inverse Fourier transform (42). Let us start by $Z^{(2)}$. Breaking the sum over $h$ into odd and even values gives immediately

$$
\begin{aligned}
Z^{(2)}= & \frac{Q-1}{2}\left\{\sum_{h_{\mathrm{even}} \neq 0} 2 Z\left(\begin{array}{c}
x=-1 \\
h
\end{array}\right)-Z\left(\begin{array}{c}
x=1 \\
h
\end{array}\right)\right. \\
& \left.-\sum_{h_{\mathrm{odd}}} Z\left(\begin{array}{c}
x=1 \\
h
\end{array}\right)+Z\left(\begin{array}{c}
x=-1 \\
0
\end{array}\right)\right\} .
\end{aligned}
$$

As far as $Z^{(1)}$ is concerned, its analysis is more difficult since one has to take into account the whole arithmetic properties of the spins $h, h^{\prime}$. For integers $p_{1}, \ldots, p_{k}$, $n_{1}, \ldots, n_{k}$ introduce the expression

$$
\left\langle\cos \frac{2 \pi}{\mathscr{N}^{\cdot}} p_{1}^{n_{1}} \ldots p_{k}^{n_{k}}\right\rangle_{c}=\sum_{0 \leq m_{\imath} \leq \inf \left(n_{\imath}, 1\right)}(-)^{\sum m_{\imath}} \cos \left(\frac{2 \pi}{\mathscr{N}^{\prime}} p_{1}^{n_{1}-m_{1}} \ldots p_{k}^{n_{k}-m_{k}}\right),
$$

then the contribution of a given sector $h \neq 0$ is seen to be

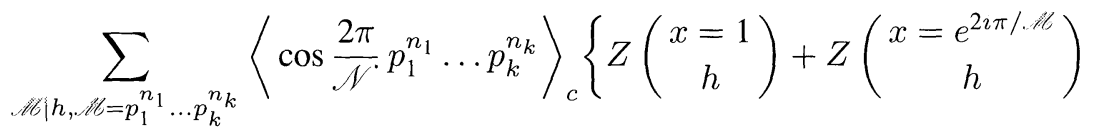

$$
\begin{aligned}
& \left.+Z\left(\begin{array}{c}
x=e^{4 i \pi / \mathscr{K}} \\
h
\end{array}\right)+\ldots+Z\left(\begin{array}{c}
x=e^{2 i \pi(\mathscr{M}-1) / \mathscr{C}} \\
h
\end{array}\right)\right\} .
\end{aligned}
$$

It thus decomposes onto generically irreducible representations. From $h=0$ one just gets $Z\left(\begin{array}{c}x=q^{2} \\ 0\end{array}\right)$, which is trace over a reducible representation. However this trace has to be combined with a trace over $Z\left(\begin{array}{l}x=1 \\ h=1\end{array}\right)$ that comes with multiplicity $Q-2$ in $Z^{(1)}$ and $-(Q-1)$ in $Z^{(2)}$ (we used the symmetry $h \rightarrow-h$ ). So on the whole we get trace over the irreducible $\varrho_{n}\left(\begin{array}{l}1 \\ 0\end{array}\right)$.

We therefore conclude that the representations of $T_{\hat{A}_{n}}(Q)$ appearing in the Potts model case are $\varrho_{n}\left(\begin{array}{l}1 \\ 0\end{array}\right)=\hat{C}_{n, 0}$ (with multiplicity one), $\varrho_{n}^{\prime}\left(\begin{array}{c}x=-1 \\ 0\end{array}\right)=\hat{C}_{n, 1}$ 
(with multiplicity $Q-1$, as expected for the order parameter), $R_{n}\left(\begin{array}{c}x=e^{2 \imath \pi k / \mathscr{M}} \\ h\end{array}\right)$ for $k=1, \ldots, \mathscr{M}-1$ and $\mathscr{C} \mid h, h$ arbitrary (but limited for a given $n$ ).

Let us now discuss multiplicities. For the case $h=2$ we get $R_{n}\left(\begin{array}{l}x=1 \\ h=2\end{array}\right)$, $R_{n}\left(\begin{array}{c}x=-1 \\ h=2\end{array}\right)$. The first one comes with multiplicity $Q^{2}-4 Q+2$ in $Z^{(1)}$ and $-Q+1$ in $Z^{(2)}$, adding to $Q^{2}-5 Q+3$. The second comes with $Q^{2}-5 Q+4$ in $Z^{(1)}$ and $2 Q-2$ in $Z^{(2)}$, adding to $Q^{2}-3 Q+2$. In general the representation $R_{n}\left(\begin{array}{c}x=1 \\ h\end{array}\right)$ arises with multiplicity $P_{2 h}-Q+1$. The representation $R_{n}\left(\begin{array}{c}x=-1 \\ h\end{array}\right)$ that arises only for $h$ even has multiplicity $P_{2 h}-P_{2}+2 Q-2$. All other representations $R_{n}\left(\begin{array}{c}x=e^{2 i \pi \mathscr{P} / \mathscr{Q}} \\ h\end{array}\right)(\mathscr{P}<\mathscr{Q}$ coprimes $)$ arise with the multiplicity, if we write the decomposition in prime factors $\mathcal{Q}=p_{1}^{l_{1}} \ldots p_{k}^{l_{k}}, h=p_{1}^{n_{1}} \ldots p_{k}^{n_{k}}$,

$$
\sum_{l_{i} \leq m_{i} \leq n_{i}} 2\left\langle\cos \frac{2 \pi}{\mathscr{N}} p_{1}^{m_{1}} \ldots p_{k}^{m_{k}}\right\rangle_{c}
$$

These can be rewritten as polynomials in $Q$, with no very illuminating forms however. The multiplicity of $R_{n}\left(\begin{array}{l}x \\ h\end{array}\right)$ is independent of $n$ for $n$ large enough, as in the $A_{n}$ and mean field case. In those cases the result is a consequence of Theorem 1. Here this theorem does not hold.

\section{Arbitrary Graphs and the Full Case}

\subsection{Examples}

Let us discuss a few examples. Consider first the Daisy graph $G=D_{4}$. As usual we start with $I_{0}$ and generate $T I_{0}$ by acting with the generators. Choose the convention that the central node in such a Daisy graph is associated with $U_{n}$. for $n-1$ legs. Then list the words

$$
\begin{array}{cccc}
I_{0} & & & \\
U_{14} I_{0} & U_{24} I_{0} & U_{34} I_{0} & \\
U_{14} U_{24} I_{0} & U_{14} U_{34} I_{0} & U_{24} U_{34} I_{0} & \\
U_{4 .} U_{14} U_{24} I_{0} & U_{4 .} U_{14} U_{34} I_{0} & U_{4 .} U_{24} U_{34} I_{0} & U_{14} U_{24} U_{34} I_{0} \\
U_{34} U_{4} U_{14} U_{24} I_{0} & U_{24} U_{4 .} U_{14} U_{34} I_{0} & U_{14} U_{4 .} U_{24} U_{34} I_{0} & U_{4 .} U_{14} U_{24} U_{34} I_{0}
\end{array}
$$

The basis truncates at this point because the relations collapse all longer words onto these. Therefore the ideal $T I_{0}$ is finite dimensional $\left[\right.$ and $\operatorname{dim}\left(T I_{0}\right)=\operatorname{dim}\left(D_{G} I_{0}\right)=$ $\left.\operatorname{dim}\left(D_{\underline{n}} I_{0}\right)\right]$. 
Consider now the graph $G=D_{5}^{(1)}$ with the incidence matrix

$$
\left(\begin{array}{llllll}
0 & 1 & 0 & 0 & 0 & 0 \\
1 & 0 & 1 & 0 & 1 & 0 \\
0 & 1 & 0 & 0 & 0 & 0 \\
0 & 0 & 0 & 0 & 1 & 0 \\
0 & 1 & 0 & 1 & 0 & 1 \\
0 & 0 & 0 & 0 & 1 & 0
\end{array}\right)
$$

Rather than generate the whole basis, we simply note that the following set of words does not collpase

$$
\left(I_{0} U_{23} U_{25} U_{45} U_{2 .} U_{5} U_{12} U_{25} U_{56} U_{2 .} U_{5 .} U_{23} U_{25} U_{45} U_{2 .} U_{5} U_{12} U_{25} U_{56} I_{0}\right)^{k}
$$

so the algebra is infinite dimensional. How can we characterize such non-collapsing words? If we draw the connectivity diagram of the above word on $G \times \mathbb{Z}$, the interior of each bracket is made of two interlocked loops: a Hopf link. In the diagram algebra, these would collapse to give two factors of $Q$. But in the Full algebra case there is no relation to simplify such a word. Recall that in the $\hat{A}$ case similar words that could not be collapsed corresponded to non-contractible loops.

Returning to $G=D_{3}$ consider now the $h=1$ sector,

$$
\begin{aligned}
& I_{1} \\
& U_{14} I_{1} \\
& U_{24} I_{1} \\
& U_{14} U_{24} I_{1} \quad U_{14} U_{34} I_{1} \quad U_{24} U_{34} I_{1} \quad U_{4} U_{24} I_{1} \\
& U_{4} U_{14} U_{24} I_{1} \quad U_{4} U_{14} U_{34} I_{1} \quad U_{4 .} U_{24} U_{34} I_{1} \quad U_{14} U_{24} U_{34} I_{1} \\
& U_{24} U_{4} U_{34} I_{1} \quad U_{34} U_{4} U_{14} I_{1} \quad U_{34} U_{4} U_{24} I_{1} \quad U_{14} U_{4} U_{34} I_{1} \\
& U_{14} U_{34} U_{4} U_{24} I_{1} \\
& U_{34} U_{4} U_{14} U_{24} I_{1} \\
& U_{4} U_{14} U_{24} U_{34} I_{1} \\
& U_{4 .} U_{14} U_{34} U_{4} U_{24} I_{1} \\
& U_{14} U_{24} U_{4} U_{34} I_{1} \\
& U_{24} U_{4} \cdot U_{14} U_{34} I_{1} \\
& U_{34} I_{1} \\
& U_{4} U_{34} I_{1} \quad U_{4} U_{14} I_{1} \\
& U_{14} U_{4} \cdot U_{24} I_{1} \\
& U_{24} U_{4} U_{14} I_{1} \\
& U_{24} U_{34} U_{4} U_{14} I_{1} \text {. } \\
& U_{14} U_{4} U_{24} U_{34} I_{1} \\
& U_{4 .} U_{14} U_{24} U_{4} U_{34} I_{1} \\
& U_{4 .} U_{24} U_{34} U_{4} U_{14} I_{1} \\
& U_{34} U_{4} U_{14} U_{24} U_{4} U_{34} I_{1} \\
& U_{14} U_{4} U_{24} U_{34} U_{4} U_{14} I_{1} \\
& U_{2 .} U_{14} U_{4} U_{24} U_{34} U_{4} U_{14} I_{1}
\end{aligned}
$$

As in the list in (50) the table truncates here. The total dimension is 36 (cf. the 4 case, the state $\left((123)(4)^{\prime}\right)$ cannot be reached from $\left.I_{1} \sim\left((1)(2)(3)(4)^{\prime}\right)\right)$. However, as we will see in the next section the $h=2$ sector is infinite dimensional.

\subsection{On the Characterization of Irreducible Representations}

Consider the $h=0$ sector. We may associate words in the ideal $T I_{0}$ with certain elements of the universal set of $G \times \mathbb{Z}$ (as we do in $D_{G}$, but here the equivalence relations are different). Words are equivalent if their graphs drawn on $G \times \mathbb{Z}$ are related by the following moves: 1 . Contraction of cul-de-sacs and removal of points, up to factors of $Q$, 
2. Deformations of the graph which take a segment to a segment without touching another segment.

Note that such a deformation may take an empty disconnected loop to a line and hence remove it at cost a factor of $Q$. To establish these moves note that objects such as cul-de-sacs and loops may be embedded in $A_{n} \times \mathbb{Z}$, where the moves are allowed by the relations.

The quotient relations required to make the ideal $T I_{0}$ finite dimensional must tell us how to deal with each type of object drawn on $G \times \mathbb{Z}$ distinct under the relations above. Suppose such an object $K$ is not equivalent to the empty graph. Then formally $\left(K I_{0}\right)^{k}$ gives a new element of the ideal for each higher integer $k$. On the other hand $T I_{0}\left(K I_{0}\right)^{k}$ is an invariant subspace of $T I_{0}$ (cf. the $\hat{A}_{n}$ case), so the way to deal with this is to introduce the quotient relation

$$
I_{0} K I_{0}=\alpha_{K} Q^{n(K)} I_{0},
$$

where $\alpha_{K} \in \mathbb{C}$ and $n(K)$ is the number of components in $K$. There is no constraint on $\alpha_{K}$ forced by the relations, since $I_{0}$ forms an impenetrable barrier (cf. the relations and the cases $A_{n}$ and $\hat{A}_{n}$ ).

Relations of this kind are not in general sufficient to produce finite dimensional ideals. However, the additional relations required to make a finite dimensional ideal do not seem to give rise to continuous parameters. Such additional relations generate still further relations which constrain the value of any parameter introduced.

The simplest example of both these features is provided by $D_{4}^{(1)}$. The normal 52 elements basis of $D_{G} I_{0}$ is extended by the non-collapse of words like

$$
\left(U_{5} U_{35} U_{15} U_{5} U_{25} U_{45}\right)^{k} I_{0}
$$

Accordingly the relations

$$
I_{0} U_{35} U_{15} U_{5 .} U_{25} U_{45}\left(U_{5} U_{35} U_{15} U_{5 .} U_{25} U_{45}\right) I_{0}=\alpha_{K} Q^{2} I_{0},
$$

which can be represented graphically as

$$
I_{0}(\text { Hopf Link }) I_{0}=\alpha_{K} I_{0}(\circ \circ) I_{0}
$$

may be introduced with no restriction on $\alpha_{K}$. In the other quotient required to get a finite dimensional ideal (and consistent with $D_{G}$ - i.e. taking connectivity to identical connectivity)

$$
\left(U_{5 .} U_{35} U_{15} U_{5 .} U_{25} U_{45}\right)^{k+2} I_{0}=z\left(U_{5 .} U_{35} U_{15} U_{5 .} U_{25} U_{45}\right)^{2} I_{0},
$$

we are forced to take $z=Q^{k}$ for consistency with the action of $U_{35} U_{15} U_{25} U_{45}$. The $D_{G} I_{0}$ quotient is the case $k=-1$, for which $\alpha_{K}$ is also determined by the relations.

The smallest representation which supports the quotient (56) (together with its images under $\mathbb{Z}_{4}$ which imbed the Hopf link into $G \times \mathbb{Z}$ in ways that are different with respect to the moves) has dimension 301. This is the case $k=1$.

Let us give a more interesting example. We will construct a free parameter quotient associated to the trefoil knot for $G$ the Daisy with 6 petals,

$$
\begin{aligned}
I_{0} U_{67} U_{57} U_{7} U_{17} U_{47} U_{7 .} U_{67} U_{27} U_{7 .} U_{37} U_{17} U_{6 .} U_{7 .} U_{67} U_{27} \\
\quad U_{7 .} U_{1 .} U_{17} U_{37} U_{7 .} U_{67} U_{47} U_{7 .} U_{17} U_{57} I_{0} \\
=\alpha_{\text {tref }} Q I_{0} .
\end{aligned}
$$




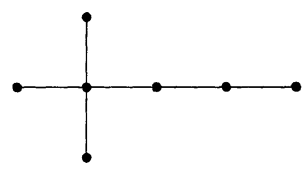

Fig. 12. A graph supporting the trefoil knot

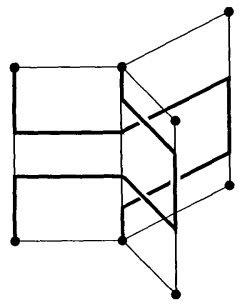

Fig. 13. A braid can be drawn on $D_{4} \times \mathbb{Z}$

The best way to visualize this is to construct a piece of paper with section $G$ and imbed the knot in this "oregamoid" such that each sheet carries at most one piece of string at a time. Incidentally the graph in Fig. 12 will also support the trefoil knot with the long leg carrying up to three lines at once.

These examples can be generalised to give

Proposition 19. There are at least as many free parameters associated to the ideal $T_{G} I_{0}$ as links embeddable in $G \times \mathbb{Z}$.

In physical terms, connectivities carry information. For instance the case $h=1$ describes spin spin correlations. The $h=0$ sector may be associated to the partition function. When we consider the $h>0$ sectors therefore we must keep track of information at the boundary of the system and hence in the present language move from links to tangles. For example on the Daisy with 5 petals the "Staffordshire Knot" (the tangle generated by opening one arc of the trefoil) gives

$$
\begin{aligned}
& I_{1} U_{56} U_{6 .} U_{16} U_{46} U_{6 .} U_{56} U_{26} U_{6 .} U_{36} U_{16} U_{5} U_{6 .} U_{56} U_{26} U_{6 .} U_{1 .} U_{16} U_{36} U_{6 .} U_{56} U_{46} U_{6 .} U_{16} I_{1} \\
& \quad=\alpha_{\text {Staff }} Q I_{1}\left(\bmod T I_{0} T\right) .
\end{aligned}
$$

Since for any $G$ other than $A_{n}$ two strands can be braided twice on $G \times \mathbb{Z}$ we have Proposition 20. All algebras $T_{G}\left(G \neq A_{n}\right)$ are infinite dimensional.

For instance for $G=D_{4}$ (see Fig. 13),

$$
b=I_{2} U_{41} U_{4} U_{43} U_{42} U_{3} U_{4 .} U_{43} U_{41} U_{4} U_{42} I_{2}
$$

is braided once, so $b^{2}=\alpha I_{2}$ is a legitimate quotient.

\section{Remarks}

\subsection{Q Non-Generic}

Until now we considered only exceptional values of the free parameters. Let us now consider exceptional values of $Q$. If there are free parameters, this is only interesting at their exceptional values (see e.g. $\mathrm{Gr}_{2.0}$ ). 
This has been understood in details for $A_{n}[2,6]$. The non-generic values in that case are given by

$$
Q=4\left(\cos \frac{\pi p}{q}\right)^{2}
$$

where $p, q$ are coprime integers. The case $p=1$ is usually referred to as Beraha numbers. The continuum limit of the corresponding ABF [23] models is given by a "minimal" conformal field theory [24] which is unitary in the $p=1$ case.

The non-generic $Q$ values in the $\hat{A}_{n}$ case are identical, as can be expected since the difference with $A_{n}$ is merely a "boundary effect."

In the mean field case preliminary study shows at least all integer $Q$ are nongeneric, and it seems likely that there are no other.

In the $A_{n}, \hat{A}_{n}$ cases, the maximum non-generic value of $Q(60)$ is $Q=4$, which coincides with the boundary between second and first order phase transitions. As we " increase the dimension" to the mean field case $G=\underline{n}$ these two numbers move therefore in opposite directions. Indeed in the mean field case the boundary between second and first order phase transitions is now at $Q=2$ while, as mentioned above, the set of non-generic $Q$ is unbounded.

A more complete study of the non-generic $Q$ case for $\hat{A}_{n}$ and $\underline{n}$ will appear elsewhere. ${ }^{*}$

In two dimensions, relations between $Q$ non-generic values and colouring problems have been observed [25]. These relations seem to extend to the mean field case.

\subsection{The General $D_{\text {Daisy }}$}

The dimensions of the irreducible representations for the general Daisy case can be computed. The numbers themselves are not very illuminating. However we note that $D_{G} I_{0}$ has the same dimension as the complete graph case (with same number of vertices). Therefore the asymptotic rate of growth of dimensions is unbounded.

\subsection{Conclusion}

Despite the difficulty of the higher dimensional case, such as $G=A_{n} \times A_{n}$, the study of algebraic properties seems an interesting alternative to the search for integrable systems. Among the important questions amenable with existing techniques (or computers) are the determination of dimensions of generically irreducible representations, and hence exceptional $Q$ values. A warm up case would be $G=A_{n} \times A_{2}$.

Acknowledgements. P.P.M. would like to thank the UK SERC, City University (London) and the Nuffield Foundation for support, and B. Westbury for useful conversations. This work was supported by the Packard Foundation and DOE Contract DE-AC02-76ERO3075.

* P. Martin, H. Saleur, preprints USC-93-009 and USC-93-010 


\section{References}

1. Zamolodchikov, A.B.: Commun. Math. Phys. 79, 489 (1981); Baxter, R.J.: In: Integrable Systems in Statistical Mechanics. Rasetti, M. et al. (eds.) Singapore: World Scientific 1985; Frenkel, I., Moore, G.: Simplex equations and their solutions. Preprint YCTP-P14-90; Saito, M., Carter, J.S.: J.K.T.R. 1, 207 (1992)

2. Martin, P.P.: Potts models and related problems in statistical mechanics. Singapore: World Scientific, 1991

3. Saleur, H.: Nucl. Phys. B 360, 219 (1991)

4. Levy, D.: Int. J. Mod. Phys. A 6, 5127 (1991)

5. Temperley, H.N.V., Lieb, E.: Proc. Roy. Soc. London A 322, 251 (1971)

6. Goodman, F., De la Harpe, P., Jones, V.: Coxeter graphs and towers of algebras. Publications of MSRI, Berlin, Heidelberg, New York: Springer 1980

7. Baxter, R.: Exactly solved models in statistical mechanics. New York: Academic Press 1982

8. Pasquier, V.: Nucl. Phys. B 285, 162 (1987)

9. Pasquier, V., Saleur, H.: Nucl. Phys. B 330, 523 (1990)

10. Martin, P.P.: Pub. RIMS Kyoto Univ. (1989)

11. Kogut, J.: Rev. Mod. Phys. 51, 659 (1979)

12. Schultz, T.,D., Mattis, D.C., Lieb, E.H.: Rev. Mod. Phys. 36, 856 (1964)

13. Cardy, J.: In: Phase transitions and critical phenomena. Vol. 11, Domb, Lebowitz (ed.) New York: Academic Press

Date, E., Jimbo, M., Miwa, T., Okado, M.: Phys. Rev. B 35, 2105 (1987)

Itoyama, H., Thacker, H.B.: Phys. Rev. Lett. 58, 1395 (1987)

14. Martin, P.P.: Temperley Lieb algebras for non planar statistical mechanics - the partition algebra. Preprint YCTP-P34-92, to appear in JKTR

15. Liu, C.L.: Introduction to combinatorial mathematics. New York: McGraw-Hill 1968

16. Cline, E., Parshall, B., Scott, L.: J. Reine A. Math. 391, 85 (1988) and references therein

17. Blote, H., Nightingale, M.P.: Physica 112A, 405 (1982)

18. Westbury, B.W.: Univ. Manchester Ph.D. thesis, 1989

19. Hamermesh, M.: Group Theory. Oxford: Pergamon 1962

20. Robinson, G. de B.: Representation theory of the symmetric group. Toronto: Toronto University Press 1961

21. Kauffman, L.: On Knots. Princeton, NJ: Princeton University Press 1987

22. Di Francesco, P., Saleur, H., Zuber, J.B.: J. Stat. Phys. 49, 87 (1987)

23. Andrews, G.E., Baxter, R.J., Forrester, P.J.: J. Stat. Phys. 35, 193 (1984)

24. Belavin, A., Polyakov, A., Zamolodchikov, A.: Nucl. Phys. B 241, 33 (1984)

25. Saleur, H.: Commun. Math. Phys. 132, 657 (1990); Kauffman, L., Saleur, H.: Commun. Math. Phys. 152, 565 (1993)

Communicated by N. Yu. Reshetikhin 